\title{
OFDM versus Single-Carrier Transmission for 100 Gbps Optical Communication
}

\author{
Alan Barbieri, Giulio Colavolpe, Member, IEEE, Tommaso Foggi, Enrico Forestieri, Member, IEEE, and
} Giancarlo Prati, Fellow, IEEE

\begin{abstract}
We analyze the orthogonal frequency division multiplexing (OFDM) technique in long-haul next generation optical communication links and compare it with the well-established single-carrier (SC) data transmission using high-level modulation formats and coherent detection. The analysis of the two alternative solutions is carried out in the $100 \mathrm{Gbps}$ scenario, which is commonly considered to be the next upgrade of existing optical links, with special emphasis on quaternary phase-shift keying (QPSK) modulations. The comparison between OFDM and SC takes into account the main linear and nonlinear impairments of the optical channel, e.g., group velocity dispersion (GVD), polarization mode dispersion (PMD), self-phase modulation (SPM), cross-phase modulation (XPM), and four-wave mixing (FWM), as well as the phase noise due to transmit and receive lasers, their relative frequency offset, other synchronization aspects, the overall complexity, the power and spectral efficiency, and the technological constraints.
\end{abstract}

Index Terms-Cross-phase modulation (XPM), four-wave mixing (FWM), group velocity dispersion (GVD), optical coherent transmission systems, orthogonal frequency division multiplexing (OFDM), phase-shift keying (PSK), polarization mode dispersion (PMD), self-phase modulation (SPM), single-carrier transmissions.

\section{INTRODUCTION}

I $\mathrm{N}$ the last decade, the huge growth of traffic on deployed optical communication networks pushed the demand for a more effective exploitation of the fiber channel capacity. The next generation optical links are going to carry $100 \mathrm{Gbps}$ per wavelength [1], [2], since the increased traffic in IP-based networks is going to make the $100 \mathrm{~Gb}$ Ethernet $(100 \mathrm{GbE})$ the future choice for high-speed long-haul optical communications.

OFDM is a well-established transmission technique [3], [4] that stands out nowadays as a standard for many wireless [5] and wired [6] communications. Its success mainly depends on its capacity to effectively transform a time-dispersive channel

Manuscript received March 31, 2010; revised May 18, 2010, June 11, 2010; accepted June 16, 2010. Date of publication June 28, 2010; date of current version August 18, 2010. The paper will be presented in part at the IEEE International Conference on Communications (ICC'10), Capetown, South Africa, May 2010. This work was supported by Ericsson $A B$.

A. Barbieri was with University of Parma, Dipartimento di Ingegneria dell'Informazione, I-43100 Parma, Italy. He is now with Qualcomm Corporation R\&D, Qualcomm Inc., San Diego, CA 92121 USA.

G. Colavolpe is with University of Parma, Dipartimento di Ingegneria dell'Informazione, I-43100 Parma, Italy.

T. Foggi is with CNIT Research Unit, I-43100 Parma, Italy.

E. Forestieri and G. Prati are with Scuola Superiore Sant'Anna, I-56124 Pisa, Italy.

Digital Object Identifier 10.1109/JLT.2010.2055041 into a set of independent and interference-free subchannels, so as to allow the use of symbol-by-symbol (SbS) detection at the receiver, rather than a complex maximum likelihood sequence detector based on the Viterbi algorithm. Furthermore, in the presence of an amplitude-selective frequency response, as in wireless communications with multipath propagation, OFDM achieves Shannon capacity through suitable power allocation and bit loading, provided that the transmitter knows the channel response, e.g., if a feedback channel is available [7], [8].

Motivated by these features, optical OFDM has been recently investigated with both direct detection (DD-OFDM) [9] and coherent detection (CO-OFDM) [10]. The former allows for a simpler receiver structure, but has a worse energy and spectral efficiency, making it more suitable for cost-effective short reach applications [11], whereas the latter features superior performance in long-haul high-data-rate transmissions, similar to single-carrier (SC) modulation formats-for these reasons only coherent OFDM will be considered in this paper.

High-speed optical coherent communication systems exhibit several specific issues which may reduce the effectiveness of OFDM with respect to $\mathrm{SC}$ formats. For instance, in the presence of phase noise and residual uncompensated carrier frequency offset, which are always present in high-speed optical communication systems [12], intercarrier interference (ICI) arises among the OFDM subcarriers to such an extent that, if not compensated, might lead to a remarkable performance degradation [13]. On the other hand, ICI compensation algorithms are computationally quite demanding, and their implementation is not feasible in such high-speed optical communication systems. A possible alternative is the use of pulseshape techniques, in which suitably designed pulses, with good time and frequency localization properties, are employed with OFDM with the aim of increasing its robustness to time-varying impairments (see, e.g., [14], [15], and references therein). However, this technique is nowadays not considered in optical communications for the technological problems related to the generation of arbitrary pulses at the transmitter. Furthermore, the large peak-to-average ratio (PAR) of OFDM signals is the reason for their large sensitivity to nonlinearities in dispersion-managed systems [16], [17]. Last, but not least, OFDM exhibits several energy and spectral overheads, such as the overhead due to the cyclic prefix (CP), and some technological drawbacks, such as the requirement of analog-to-digital and digital-to-analog converters with an higher resolution than that required in SC systems. On the other hand, SC transmissions require a fractionally-spaced two-dimensional linear equalizer to compensate for linear impairments (GVD and PMD) [18]. For a single carrier system, 
this equalizer represents the most computational demanding and technological challenging block.

Motivated by the above considerations, the aim of this paper is to carry out an extensive investigation of the practical potential of OFDM for use over optical communication links at 100 Gbps, by means of both analytical characterization and computer simulations. Specifically, we will compare, in terms of performance, complexity, and overall state-of-the-art feasibility, an OFDM communication system with the single-carrier communication scheme described in [18], for which a parallel and computationally efficient implementation is also discussed here for better comparison with OFDM. In both cases we will assume the adoption of polarization multiplexing and a quaternary phase-shift keying (QPSK) modulation format, as this is going to be the format used in long-haul $100 \mathrm{Gbps}$ links. We partly anticipate here the main conclusion that we will draw after this extensive analysis: although OFDM certainly has a lot of merits, and in fact has been selected for several wireless communication standards, in the considered high-speed optical communication channel and with the optical and electronic devices available today, it does not compare favorably to SC techniques, mainly because the latter do not require digital-to-analog converters at the transmitter. However, for higher transmission rates, SC systems could be forced to use still higher modulation formats, which would increase the transmitter complexity, contrarily to the OFDM case. A paper with similar aim has been recently published [11]. Conclusion are quite similar, although we show here novel and more detailed numerical results.

The paper is organized as follows. In Section II we present a thorough literature analysis of both SC and OFDM systems for optical communications. Then, in the following three sections, we describe the three main impairments faced by a high-speed fiber-optic system, namely linear time-invariant distortions, linear time-varying distortions (mainly phase noise and carrier frequency offset), and Kerr-type nonlinear distortions, along with their effects on communication and the ways to overcome them. Technological drawbacks, computational complexity analysis, and an analysis of the sources of energy and spectral overheads for both techniques are presented in Section VI. Numerical results are reported in Section VII and, finally, considerations about the use of OFDM systems for future high-speed optical communications will be presented in Section VIII.

Notation: We denote vectors and matrices with bold and calligraphic-bold symbols, respectively. Whether a symbol refers to a deterministic or a random quantity, is either clear from the context, or explicitly specified. Lowercase letters are used for time domain quantities, whereas capital letters are reserved for frequency-domain ones.

\section{SYSTEM DESCRIPTION}

We consider long-haul optical transmissions using polarization multiplexing. In these systems, two independent data streams are sent on two orthogonal states of polarization (SOPs). Provided that a proper front-end is employed at the receiver to extract two orthogonal polarizations, the optical channel can be seen as a two-input two-output channel which, in the linear regime, is described by a $2 \times 2$ transfer matrix

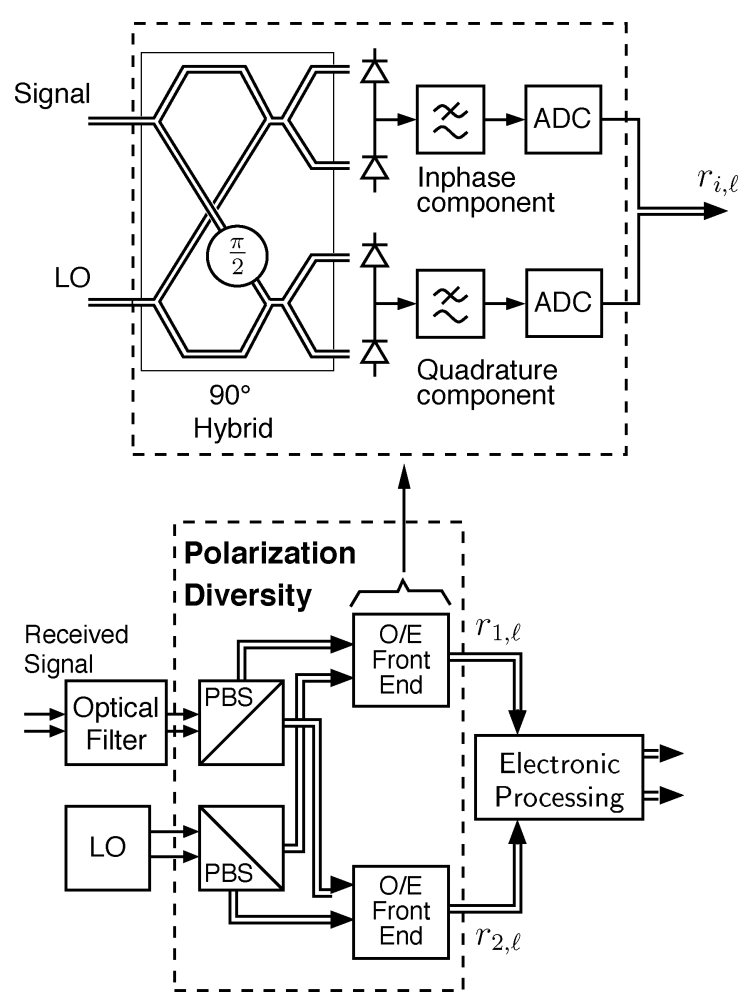

Fig. 1. Receiver structure.

(Jones matrix). We consider the receiver as composed of an analog opto-electronic $(\mathrm{O} / \mathrm{E})$ front end, devoted to signal demodulation and conversion from the optical to the electrical domain, and a digital part devoted to electronic processing (see Fig. 1). After a preliminary optical filtering, two orthogonal SOPs are split through a polarization beam slitter (PBS). They are then separately combined with the optical field of a local oscillator laser (LO) in a $2 \times 490^{\circ}$ hybrid [19] and detected with two balanced photodetectors. In this way, the two received signals, one for each SOP, are converted in the electrical domain, in practice performing a frequency conversion. Denoting by $T_{c}$ the sampling interval, the samples at time $\ell T_{c}$ of the two received signals are collected in a two-dimensional column vector $\mathbf{r}_{\ell}$. Since a frequency estimation and compensation block is required before detection for both SC and OFDM systems, we will denote by vector $\mathbf{y}_{\ell}$ the samples at its output.

\section{A. Single-Carrier (SC)}

The SC system considered in this paper is described in detail in [18]. As a discrete-time sufficient statistic is obtained through oversampling, the main component is represented by a two-dimensional fractionally-spaced linear equalizer which, as shown in [18] (where the most recent works on linear equalization for coherent optical systems are reported and analyzed), is able to perfectly compensate for GVD and PMD provided it has a sufficient number of taps. The adoption of the asynchronous strategies for detection and for the adjustment of the equalizer taps described in [18] allows also to remarkably increase the robustness to phase noise. In the following, we will denote by $L_{c}$ the number of equalizer taps and by $N$ the number of past decisions used by the asynchronous detection strategy [18], [20]. 


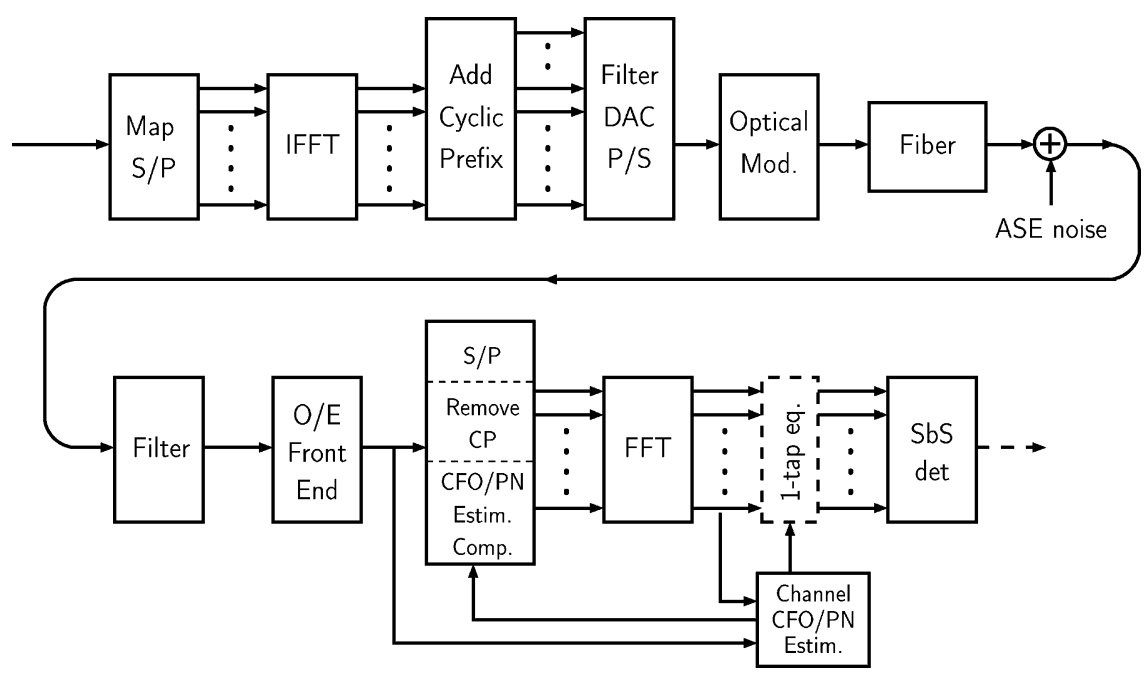

Fig. 2. Block diagram of an OFDM optical communication system.

Assuming, as in [18], that two samples per symbol interval $T$ are employed, GVD and PMD will be perfectly compensated when $2 L_{c} T$ is larger than or equal to the duration of the channel impulse response. ${ }^{1}$

Although some of the implementation aspects, such as the impact of a fixed-point implementation, have been addressed in [18], other aspects will be further investigated here in order to give a complete picture necessary for a fair comparison with optical OFDM systems. In particular, we will describe how the two-dimensional linear equalizer can be parallelized (using the overlap-save technique [21]) and also implemented in the frequency domain for complexity-saving purposes.

\section{B. $O F D M$}

Several authors recently proposed using OFDM in high-speed optical communication links as a possible alternative to standard SC modulations [22]-[24]. The block diagram of an OFDM optical communication system without polarization multiplexing is shown in Fig. 2. The extension to the case of polarization multiplexing is straightforward and can be found in [25], [26].

OFDM consists in modulating and demodulating blocks of symbols, previously mapped into a given constellation, through discrete Fourier transform (DFT), usually exploiting the efficient inverse and direct fast Fourier transform (IFFT/FFT) algorithms [27]. Therefore, symbols in each block are modulated onto subcarriers, whose orthogonality ensures that no intercarrier interference occurs, provided that the overall channel is time-invariant. Thus, the transmitted signal, neglecting phase noise and frequency offset, can be remapped at the receiver to the original constellation. It is straightforward to understand how an adjustable allocation of the transmitted signal to convenient subcarriers allows to avoid channel spectral dips, which could severely harm system performance [28]. And, in fact, ODFM with proper power allocation achieves Shannon capacity.

\footnotetext{
${ }^{1} \mathrm{~A}$ non-integer number of samples per symbol interval would reduce the number of equalizer taps but would require an interpolator after the equalizer. This aspect will be discussed later.
}

In the optical communication literature, the investigation on OFDM is motivated by its effectiveness in mitigating linear channel impairments, such as group velocity dispersion (GVD) and polarization mode dispersion (PMD), by simply setting a proper cyclic prefix overhead and using a one-tap equalizer at the receiver, and by the implicit parallelization of the processing due to the IFFT/FFT operations, which clearly represents an advantage in high-speed optical links [25]. The optical channel does not entail any changes in the principles of the OFDM technique - the use of polarization multiplexing simply implies the adoption of a couple of OFDM transmitters and a couple of FFT blocks at the receiver with a two-dimensional 1-tap equalizer [25], [26]. For this reason, most of the works published on optical OFDM deal with the experimental demonstration of different approaches and solutions to overcome technological issues [26], [29]-[31].

Through the use of virtual subcarriers, OFDM allows to easily accommodate for transmit filters with a smooth transition band [6]. It can be easily shown that the use of these virtual subcarriers is equivalent to oversampling, which is therefore easily accounted for in OFDM systems. This is a main advantage in OFDM with respect to SC schemes. With the commonly used filters in optical systems, $20 \%$ of virtual subcarriers is typically sufficient [32]. On the contrary, one of the most constraining aspects in the design of an optical OFDM system is the generation of the analog waveform at the transmitter by means of high-speed digital-to-analog converters (DACs). Nowadays, the available technology does not allow the implementation of single-band OFDM streams. The only way to implement a 100 Gbps optical OFDM transmission is therefore the parallelization of transmit (and potentially receive) architecture, so that less stringent bandwidth requirements can be managed by existing optical components. In this direction, some works have been published, addressing theoretical and experimental subcarrier multiplexing (SCM), or multi-band OFDM, [26] and orthogonal-band multiplexing (OBM) [29], [33]. Basically, the technological limit is overcome by multiplexing different OFDM sub-signals and reducing the guard-bands between sub-bands by exploiting subcarrier orthogonality between different sub- 
bands, in the same way it is exploited in a single OFDM frame, thus significantly reducing intercarrier interference. These techniques, although making a 100 Gbps OFDM transmission possible, imply an increase of hardware complexity, which should be accounted for in a fair comparison with SC transmissions. However, in the remainder of the paper a single-band OFDM transmission is assumed, as it will not affect the analysis and the main conclusions that will be carried out.

In optical communications, phase noise due to transmit and receive lasers, as well as the presence of a residual uncompensated carrier frequency offset (CFO), are expected to degrade the performance of OFDM, due to the fact that ICI arises in this scenario. A time-domain phase noise compensation, which avoids the onset of ICI, was proposed in [12], based on the transmission of an unmodulated carrier within the band occupied by the transmitted signal. If the phase noise is slow enough, ICI can be neglected even without any explicit compensation, although carrier phase must be anyway estimated [34]-[36].

The impact of fiber nonlinear impairments, such as self-phase modulation (SPM), cross-phase modulation (XPM), and fourwave mixing (FWM), has also been addressed in the literature, as it is known that, in dispersion-compensated links, the high PAR characterizing OFDM signals has a more detrimental effect on system performance than in SC transmission. In [37], [38], it was proposed to exploit a phase compensation method from [39] to mitigate the nonlinearities-induced phase distortions, whereas in [40] and [41], the effectiveness of predistortion and signal clipping at the transmitter against nonlinear impairments was investigated. Some of these techniques involve a complexity increase at the transmitter and/or the receiver. Finally, in [42] the joint processing of two polarization components in polarization division multiplexed OFDM systems for more effective SPM compensation and the impact of the dispersion map design on the performance was explored. In the presence of XPM, it was also found that conventional OFDM channel estimation schemes using time/polarization-interleaved training symbols fails, and a new chennel estimator was developed to solve this problem. In the following, we develop the analysis of the two competing solutions, as said in the introduction.

\section{TIME-INVARIANT LINEAR DiSTORTIONS}

We take into account a channel consisting of multiple spans of SMF, with amplification and dispersion compensation after each span. In the linear regime and in the absence of polarization-dependent loss, the signal is corrupted only by PMD, GVD, and amplified spontaneous emission (ASE) noise. In general, depending on the dispersion map design, there will be a non-zero residual fiber dispersion $D_{r}$ (usually expressed in $\mathrm{ps} / \mathrm{nm}$ ). We denote by $\mathcal{H}(f)$ the $2 \times 2$ Jones matrix representing the two-dimensional frequency response of the channel, accounting for both GVD and PMD and a possible constant unknown phase shift due to the phase uncertainty of the transmit and receive lasers. $^{2}$ The fiber Jones matrix is unitary, irrespective of the

\footnotetext{
${ }^{2}$ The phase noise due to the transmit and receive lasers will be taken into account later.
}

amount of residual GVD and the amount or model (1st, 2nd, or higher) of PMD [43]. Hence

$$
\mathcal{H}(f) \mathcal{H}^{H}(f)=\mathcal{H}^{H}(f) \mathcal{H}(f)=\mathcal{I}
$$

where $\mathcal{I}$ is a $2 \times 2$ identity matrix. ${ }^{3}$ We will also denote by $\mathfrak{h}_{\ell}$ the $2 \times 2$ channel impulse response matrix accounting not only for PMD and GVD but also for digital-to-analog and analog-todigital converters (DACs and ADCs), as well as transmit and receive filters, assumed, as in [18], such that no intersymbol interference (ISI) arises in the back-to-back (b2b) case. ${ }^{4}$

The low-pass equivalent of the accumulated ASE noise on two orthogonal SOPs can be modeled as a couple of independent complex noise components, each with two-sided power spectral density (PSD) equal to $N_{0}$. Denoting by $\mathbf{x}_{n}$ the vector collecting the transmitted samples on the same two orthogonal SOPs at time $n$ and assuming perfect frequency compensation, the received samples can be expressed as [18]

$$
\mathbf{y}_{n}=\sum_{\ell=0}^{L-1} \mathbf{h}_{\ell} \mathbf{x}_{n-\ell}+\mathbf{w}_{n}
$$

where the two components of $\mathbf{w}_{n}$ are independent and identically distributed complex Gaussian random variables each with mean zero and variance $\sigma^{2}=N_{0} / T_{c}$ [18], $T_{c}$ being the sampling interval. In (2), as done in [18], we assumed a finite impulse response of length $L$ samples. However, we point out that the impulse response of a fiber has, in principle, an infinite duration, although, since its energy is obviously finite, it decays to zero for increasing values of $\ell$. Hence, $L$ is chosen such that the energy of the impulse response leaking outside the range $0 \leq \ell<L$ is negligible, according to some criterion.

\section{A. Single-Carrier}

As mentioned, being a SMF a frequency-flat channel in the linear regime [see (1)], and for the above mentioned hypothesis on the transmit and receive filters, a two-dimensional linear equalizer with at least $L_{c}=L$ taps is able to perfectly compensate for GVD and PMD [18]. This means that, contrary to what happens in the case of frequency-selective wireless and wired channels, where an optimal maximum-likelihood sequence detector involves the use of a Viterbi processor, a processing with complexity linear and not exponential in $L$ is required. For the same reason, there is no need to perform non-uniform power allocation in order to achieve capacity. Hence, the main advantages of OFDM over SC do not hold in this case.

The straightforward implementation of the two-dimensional linear equalizer, however, results in a non-parallel and computationally demanding architecture even for a limited number of

\footnotetext{
${ }^{3}$ In the following $(\cdot)^{T}$ and $(\cdot)^{H}$ denote transpose and transpose conjugate, respectively.

${ }^{4}$ Rigorously, since PMD is a time-varying phenomenon, the system is linear but not time-invariant. However, due to its slowly-varying nature [44], in SC systems it will be considered time-invariant-the algorithm for the adjustment of the equalizer taps will take care of this. In OFDM systems, since the coherence time of PMD is order of magnitudes larger than any practical OFDM symbol duration [44], the channel impulse response will be considered invariant at least over an OFDM symbol and will be denoted by $\mathbf{h}_{\ell}^{(m)}, m$ being the symbol index.
} 


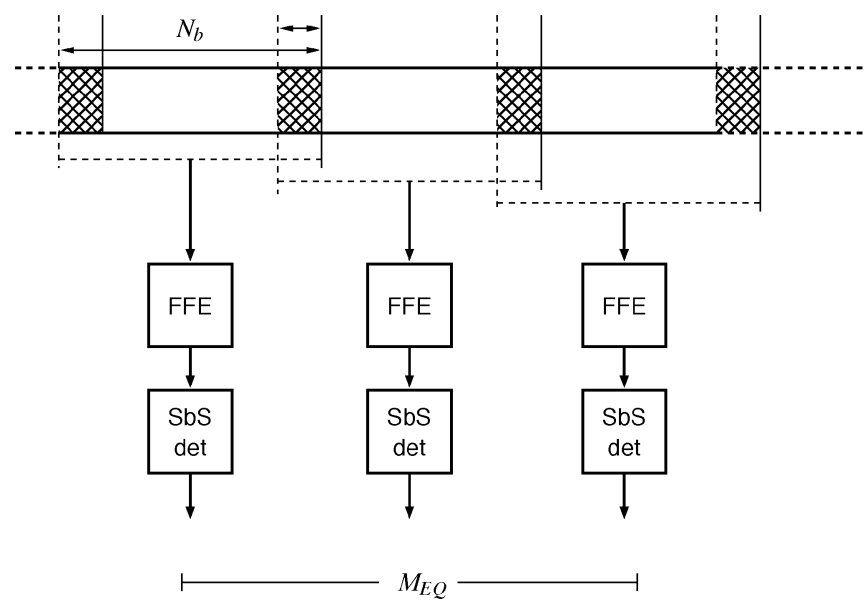

Fig. 3. Concept of a time-domain equalizer with overlap-save technique. $M_{\mathrm{EQ}}$ blocks of overlapping $N_{b}$ samples are processed by $M_{\mathrm{EQ}}$ identical two-dimensional equalizers.

equalizer taps. It is known, in fact, that the time-domain straightforward implementation of a linear convolution between the equalizer impulse response and an input sequence is not computationally convenient with respect to other, more sophisticated, methods even for a relatively short impulse response [21]. However, parallel processing and computational savings can be achieved as follows.

1) Time Domain Equalization: Equalization can still be done in the time domain, but by splitting the received data stream into overlapping subblocks, thus implementing the overlap-save technique [21], as shown in Fig. 3. Each subblock of $N_{b}$ samples is processed by one of $M_{\mathrm{EQ}}$ identical two-dimensional equalizers and overlaps by a number of samples that depends on the equalizer length $L_{c}$ and the number $N$ of past decisions used by the asynchronous detection strategy described in [18], when employed. In fact, an overlap of $L_{c}$ samples is required for the transient of the equalizer (to fill the equalizer tapped-delay-line) whereas it is possible to show, by computer simulations, that a further transient of $2 N$ symbols, thus $2 N T / T_{c}$ samples, is required by the asynchronous detection strategy to provide no performance degradation. Hence, a parallel architecture at a lower speed is obtained at the expense of hardware redundancy, latency, and a complexity increase, due to the need of a double processing of the overlapping windows. This complexity increase is related to the ratio $\left(N_{b}+L_{c}+2 N T / T_{c}\right) / N_{b}$. However, since the degree of parallelism $M_{\mathrm{EQ}}$ and the subblock length $N_{b}$ are degrees of freedom, this ratio can be kept as small as desired. Notice that no overhead in terms of bandwidth or energy is entailed and that, due to the fact that the channel is slowly varying, all equalizers can employ the same taps and adaptation can be performed on one equalizer only. In other words, no hardware redundancy is required for the circuitry necessary to update the taps. The lower speed allows also to implement interpolation techniques necessary to work with a non-integer number of samples per symbol interval (in particular with less than 2 samples per symbol interval), thus reducing the computational complexity.

2) Frequency Domain Equalization: Equalization can be performed in the frequency domain (FD) [21], [45], [46],

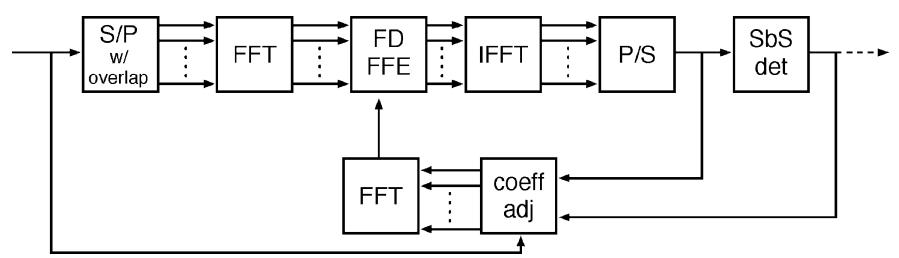

Fig. 4. Block diagram of a frequency-domain equalizer.

as shown in Fig. 4. The above mentioned subblocks of $N_{b}$ samples are transformed through FFT. Similarly, once the equalizer coefficients are transformed, equalization can be easily accomplished through a multiplication between transforms (performed by a 1-tap parallel equalizer similar to that used in OFDM receivers). The corresponding output is then transformed back again to the time domain for (asynchronous) $\mathrm{SbS}$ detection. In this FD implementation of the equalizer for SC transmissions, we have the same main hardware component blocks (those implementing FFT and IFFT) used in OFDM, but in this case they all reside at the receiver. In addition, since the equalizer coefficients are updated in the time domain, a further FFT block must be employed. This implies an increase in complexity (although very limited since the equalizer taps must be updated at a very low rate, being PMD a slowly-varying phenomenon [44]) but has the advantage that the asynchronous strategy for the adjustment of the equalizer coefficients [18] can be adopted, thus remarkably increasing the robustness against phase noise. In this case, since both OFDM and SC with FD equalization mainly employ the same hardware components, complexity will be similar. This architecture has also two other main advantages. First, very long equalizers can be implemented, thus allowing to compensate for the whole fiber dispersion (when dispersion-management is not employed) and not only for the residual one. Then, this architecture allows to manage in a simpler way a non-integer number of samples that, in a time-domain implementation would have required an explicit interpolator. In this FD implementation, interpolation could be embedded in the FD equalizer [21]. As a conclusion, this implementation architecture allows to remarkably reduce the processing complexity of the SC transmission scheme.

\section{B. $O F D M$}

OFDM is an effective way to decompose a time-dispersive channel into a set of independent and parallel subchannels [6]. Hence, when OFDM is employed and the cyclic prefix is long enough to ensure perfect compensation of the dispersion (see below), a trivial SbS detector can be used for data detection. This is the reason for the recent widespread use of OFDM in several wireless communication systems, where, as mentioned, SC transmissions with SbS detection are not able to achieve channel capacity.

We denote by $\mathbf{X}_{k}^{(m)}$ the frequency-domain complex modulation symbol associated to the $k$-th subcarrier and the $m$-th OFDM symbol, with $k=0, \ldots, N_{\mathrm{FFT}}-1$, where $N_{\mathrm{FFT}}$ is the employed FFT size. $N_{\mathrm{FFT}}$ is a design parameter and must be carefully chosen according to several criteria, which will be discussed in this and the next sections. Note that, in principle, each $\mathbf{X}_{k}^{(m)}$ could belong to a different constellation, although 
we assume that QPSK is used for all subcarriers. In fact, the optical channel being frequency-flat, the only reason for different power allocation and bit loading could be the imperfections in the frequency response of electrical and optical filters [47], not considered here. Moreover, a few side subcarriers are sometimes turned off, namely it may exist $N_{\mathrm{VC}} \geq 0$ such that $\mathbf{X}_{k}=[0,0]^{T}$, for all $k=\left(N_{\mathrm{FFT}}-N_{\mathrm{VC}}+1\right) / 2, \ldots,\left(N_{\mathrm{FFT}}+\right.$ $\left.N_{\mathrm{VC}}-1\right) / 2$.

If we denote by $N_{\mathrm{CP}}$ the length of the cyclic prefix, the timedomain samples of the discrete-time transmitted signal are

$$
\begin{gathered}
\mathbf{x}_{\left(N_{\mathrm{FFT}}+N_{\mathrm{CP}}\right) m+n}=\frac{1}{N_{\mathrm{FFT}}} \sum_{k=0}^{N_{\mathrm{FFT}}-1} \mathbf{X}_{k}^{(m)} e^{j 2 \pi k n / N_{\mathrm{FFT}}}, \\
n=0, \ldots, N_{\mathrm{FFT}}+N_{\mathrm{CP}}-1 .
\end{gathered}
$$

The continuous-time transmitted signal is obtained from the corresponding discrete-time sequence through a continuous-time pulse, which includes explicit filtering, implicit filtering carried out by the DAC and the amplifier, etc. 5 The transmitted signal also undergoes linear dispersive effects introduced by the fiber, as well as all linear effects due to filtering and analog-to-digital conversion at the receiver. As mentioned, all these effects are embedded in the time-varying matrix impulse response $\mathfrak{h}_{\ell}^{(m)}$ in (2), which may change from one OFDM symbol to the next. After CP removal, the discrete-time sequence is fed to an OFDM demodulator, whose frequency-domain complex output symbols are

$$
\begin{array}{r}
\mathbf{Y}_{k}^{(m)}=\sum_{n=N_{\mathrm{CP}}}^{N_{\mathrm{FFT}}+N_{\mathrm{CP}}-1} \mathbf{y}_{\left(N_{\mathrm{FFT}}+N_{\mathrm{CP}}\right) m+n} e^{-j 2 \pi k n / N_{\mathrm{FFT}}}, \\
k=0, \ldots, N_{\mathrm{FFT}}-1 .
\end{array}
$$

In order for OFDM to be effective in removing the intersymbol interference stemming from the dispersion (see (2)), in addition to the above mentioned hypothesis that the impulse response must vary slowly enough so that it can be assumed constant over the duration of an OFDM symbol, CP must be longer than the impulse response (i.e., $N_{\mathrm{CP}} \geq L-1$ ). In this case, combining (3), (2), and (4) it turns out that

$$
\mathbf{Y}_{k}^{(m)}=\mathcal{H}_{k}^{(m)} \mathbf{X}_{k}^{(m)}+\mathbf{W}_{k}^{(m)}
$$

where $\mathbf{W}_{k}^{(m)}$ are the frequency-domain additive noise samples (still a white Gaussian process), whereas the frequency-domain matrix channel response corresponding to the $k$-th subcarrier reads

$$
\mathcal{H}_{k}^{(m)} \triangleq \sum_{\ell=0}^{L-1} \boldsymbol{h}_{\ell}^{(m)} e^{-j 2 \pi \ell k / N_{\mathrm{FFT}}}
$$

From (5), it reads clear that the interference has been perfectly removed, and a $\mathrm{SbS}$ detection can be carried out on the fre-

\footnotetext{
${ }^{5}$ The effect of quantization of the ADC and the DAC is not included in this derivation.
}

quency-domain complex vectorial samples $\mathbf{Y}_{k}^{(m)}$ to recover the corresponding transmitted samples $\mathbf{X}_{k}^{(m)}$.

We remark that, if either one of the conditions above does not hold, (5) is no longer true and ICI appears among the subcarriers, i.e., some of the energy of each frequency-domain symbol leaks on the neighbouring subcarriers. In this case, SbS detection is no longer optimal in the maximum likelihood sense, and a large performance degradation might be expected if such receiver is still used. Interference cancellation algorithms have been proposed to cope with this situation (see, e.g., [48]-[51]). However, the computational complexity of the compensation algorithms is definitely unaffordable in the considered context, due to the large signaling rate. Hence, these approaches will not be considered here. Besides ICI, inter-OFDM-symbol interference also arises when the $\mathrm{CP}$ is shorter than the impulse response. This entails a further performance degradation if such an interference is not taken into account at the receiver.

From the above discussion, it reads clear that CP must be long enough in order to avoid interference. On the other hand, we remark that $\mathrm{CP}$ represents an overhead, since the time and energy devoted to the transmission of the $\mathrm{CP}$ are wasted information-wise. In particular, the loss in terms of energy and spectral efficiency is $N_{\mathrm{CP}} /\left(N_{\mathrm{FFT}}-N_{\mathrm{VC}}+N_{\mathrm{CP}}\right)$. In order to keep the overhead bounded to reasonable levels, it must be $N_{\mathrm{CP}} \ll N_{\mathrm{FFT}}$, but since $N_{\mathrm{CP}} \geq L-1$ for avoiding interference, we are forced to take large values of $N_{\mathrm{FFT}}$, unless very small values of residual dispersions are considered. As we will clarify in the next sections, there are several reasons to keep the value of $N_{\mathrm{FFT}}$ small. Hence, the choice of the design parameter $N_{\text {FFT }}$ is one of the most challenging problems when designing an OFDM-based communication system. Due to the non-negligible computational complexity they add, we will not consider here channel shortening equalization algorithms (see [52], [53] and references therein), whose aim is to reduce the value of $L$ so that $N_{\mathrm{CP}}$ can be reduced as well.

In the overall OFDM channel model (5), channel coefficients $\mathcal{H}_{k}^{(m)}$ are unknown at the receiver, since they depend on the random fiber dispersion. Usually, pilot symbols are periodically introduced in the transmitted signal, and used by the receiver for channel estimation and tracking. These symbols represent a further source of overhead. An alternative approach, which requires the insertion of pilot symbols at the beginning only, is based on the use of a differential encoding across subcarriers and the adoption of the detection strategy described in [20], straightforwardly extended to the two-dimensional channel model (5), after FFT at the receiver. ${ }^{6}$ In fact, a preliminary channel estimation based on pilot symbols will allow to compensate for the contribution to the channel frequency response due to GVD_-since this contribution depends on $k^{2}$, where $k$ is the subcarrier index, it cannot be compensated for by the detection strategy in [20]. On the contrary, the slowly-varying fluctuations due to PMD can be easily compensated without the need to resort to additional pilot symbols. Since the adoption of differential encoding across subcarriers and of the detection

${ }^{6}$ Differential encoding across subcarriers entails a double number of symbol errors which translates in a loss of a few tenths of $\mathrm{dB}$ in terms of signal-to-noise ratio, provided an advanced detection algorithm, such as that described in [20], is employed. 
strategy described in [20] also increases the receiver robustness in the presence of slow phase noise, it will be always considered in our simulation results.

\section{Phase NoISE AND CFO}

In coherent optical systems, transmit and receive lasers induce, on the received signal, a phase noise whose linewidth ranges from a few hundreds $\mathrm{kHz}$ for external cavity lasers to a few $\mathrm{MHz}$ for cheaper distributed-feedback lasers. At the same time, it is necessary to take into account the presence of a nonnegligible residual offset after frequency synchronization, performed in the electrical domain in the intradyne receiver to benefit from the absence of expensive and problematic synchronization optical devices such as optical phase-lock loops (PLLs). Phase noise and uncompensated CFO are the major limiting factors in coherent optical systems and, due to their similar nature, they will be discussed together in this section.

In the presence of receive phase noise and/or a residual uncompensated CFO, the $n$-th received vectorial sample reads

$$
\mathbf{y}_{n}=e^{j \theta_{n}} \sum_{\ell=0}^{L-1} \mathfrak{h}_{\ell} \mathbf{x}_{n-\ell}+\mathbf{w}_{n}
$$

where $\theta_{n}$ is the time-varying phase process, given by

$$
\begin{aligned}
\theta_{n} & =\phi_{n}+2 \pi n \Delta F T_{c} \\
\phi_{n} & =\phi_{n-1}+v_{n} .
\end{aligned}
$$

In (8), $\phi_{n}$ is the time-varying phase noise, which follows a Wiener model (namely, $\left\{v_{n}\right\}$ denote a sequence of i.i.d. zeromean Gaussian samples, whose standard deviation depends on the laser linewidth). Moreover, $\Delta F$ is the residual CFO, which is usually normalized with respect to the sampling frequency $1 / T_{c}$.

\section{A. Single-Carrier}

The effects of phase noise and an uncompensated CFO on SC optical coherent systems have been addressed in many works in the latest 20 years. We recently proposed a receiver architecture for $100 \mathrm{Gbps}$ intradyne coherent optical systems [18]. The adoption of asynchronous strategies for $\mathrm{SbS}$ detection and for the adjustment of the coefficients of the two-dimensional equalizer, not only significantly increases the robustness to phase noise, but has also the convenient side effect of increasing the robustness to an uncompensated frequency offset-errors up to $10^{-3} / T$ are tolerated. Hence, before the two-dimensional equalizer, a simple automatic frequency control (AFC) loop is sufficient to guarantee the reduction of the residual frequency offset to the desired values [18]. As shown in [18], this architecture is the most convenient one in terms of robustness to strong phase noise and computational load. Other more robust and performing algorithms could be employed [54], but at the expense of a non-negligible complexity increase. For this reason, the receiver architecture in [18] is considered here except that equalization is performed in the frequency domain for the above mentioned implementation advantages.

\section{B. $O F D M$}

In the presence of a time-varying impairment, OFDM fails to decompose the channel into parallel and independent subchannels. In particular, a time-varying channel impulse response ${ }^{7}$ destroys the orthogonality among the subcarriers, thus ICI arises [55]-[57]. In such a scenario, if countermeasures are not adopted, as discussed later, a performance degradation is unavoidable. In fact, interference acts as a further source of random noise, which, in certain circumstances, can be even dominant with respect to the additive white Gaussian noise. On the other hand, if the channel impulse response is almost constant over an OFDM symbol, ICI is certainly negligible. A well known rule of thumb, widely employed to assess the potential impact of ICI, is to evaluate the ratio between the channel coherence time, namely the time over which the channel impulse response is almost constant (according to some criterion), and the OFDM symbol duration. Larger values of this ratio entail reduced impact of the ICI.

In a high-speed optical communication system, as the one considered in this paper, there are mainly three linear timevarying distortions, namely PMD, phase noise, and CFO. While PMD usually varies slowly enough so that the resulting ICI is negligible, since its coherence time is orders of magnitude larger than any reasonable OFDM symbol duration (see Section III.B), phase noise and CFO might entail remarkable ICI due to their relatively fast speed. In this case, the received vectorial sample corresponding to the $k$-th subcarrier can be written as

$$
\mathbf{Y}_{k}=\sum_{\ell=0}^{N_{\mathrm{FFT}}{ }^{-1}} \Psi_{k-\ell} \boldsymbol{H}_{\ell} \mathbf{X}_{\ell}+\mathbf{W}_{k} .
$$

having omitted the OFDM symbol index for the sake of notation simplicity, since we assume that the $\mathrm{CP}$ is longer than the impulse response duration, and assumed that no estimation and compensation of phase noise and frequency offset is performed. The $N_{\text {FFT }}$-periodic sequence of complex scalars $\Psi_{i}$, which depends on the sequence of phases $\left\{\theta_{n}\right\}_{n=N_{\mathrm{CP}}}^{N_{\mathrm{FFT}}+N_{\mathrm{CP}}-1}$, determines the amount of ICI. In particular, it turns out that

$$
\Psi_{i}=\frac{1}{N_{\mathrm{FFT}}} \sum_{n=N_{\mathrm{CP}}}^{N_{\mathrm{FFT}}+N_{\mathrm{CP}}-1} e^{j \theta_{n}} e^{-j 2 \pi i n / N_{\mathrm{FFT}}} .
$$

From (10) it reads clear that, in order to ensure absence of ICI, namely $\Psi_{i}=\delta_{i}$ (being $\delta_{i}$ the Kronecker delta), the phase process $\theta_{n}$ must be constant. On the contrary, (9) can be rewritten as

$$
\mathbf{Y}_{k}=\Psi_{0} \mathcal{H}_{k} \mathbf{X}_{k}+\mathbf{I}_{k}+\mathbf{W}_{k}
$$

where $\mathbf{I}_{k}=\sum_{\ell \neq k} \Psi_{k-\ell} \mathcal{H}_{\ell} \mathbf{X}_{\ell}$ represents the interference.

The impact of a residual CFO on the ICI can be easily characterized analytically from (10). In the absence of phase noise, namely when $\phi_{n}$ is constant in (8), it turns out that (we show only the magnitude of the ICI coefficients)

$$
\left|\Psi_{i}\right|=\frac{1}{N} \frac{\sin \left(\pi \Delta F T_{c} N_{\mathrm{FFT}}\right)}{\sin \left(\pi\left(\Delta F T_{c}-i / N_{\mathrm{FFT}}\right)\right)} .
$$

${ }^{7}$ In this Section we assume a linear channel, thus neglecting possible nonlinear time-varying effects which will be taken into account in Section V. 


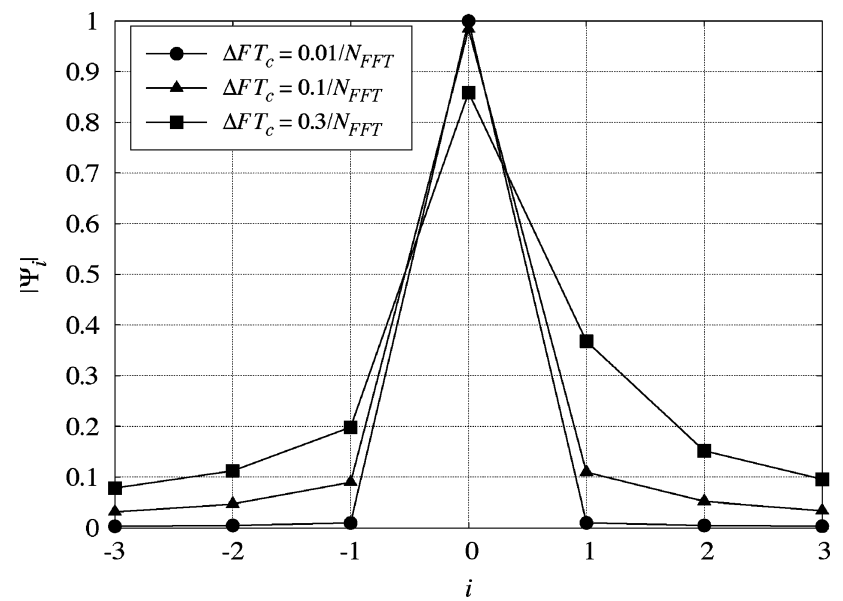

Fig. 5. Magnitude of the coefficients $\Psi_{i}$, for $N_{\mathrm{FFT}}=256$ and three values of the normalized CFO $\triangle F T_{c}$.

In Fig. 5 we show the magnitude of $\Psi_{i}$, for $i=0, \pm 1, \pm 2, \pm 3$, and for three values of $\Delta F T_{c}$.

Clearly, the larger the CFO, the higher the amount of energy leaking from a subcarrier to the neighbouring subcarriers. Hence, it is of paramount importance to ensure that the residual $\mathrm{CFO}$ after the AFC is order of magnitudes smaller than the frequency separation between the subcarriers, i.e., $1 / N_{\mathrm{FFT}}$. In other words, when OFDM is employed, either a more robust $\mathrm{AFC}$ is used, able to reduce the residual $\mathrm{CFO}$ to the required values, or the OFDM symbol duration must be reduced, so as to increase the frequency separation among the subcarriers.

As opposed to the case of CFO, whose impact can be easily studied in closed form, an analytical characterization of the ICI in the presence of random phase noise is much harder. Although some authors faced this problem (see, e.g., [13], [58], [59]), numerical simulations are usually unavoidable in this case. From (10) and (11) it turns out that, in the presence of a time-varying phase, the magnitude of the signal coefficient $\left|\Psi_{0}\right|$ decreases, while the magnitude of the interference coefficients $\left|\Psi_{i}\right|, i \neq 0$, grows. We evaluated the cumulative distribution function (cdf) of $\left|\Psi_{i}\right|$ (namely, $P\left\{\left|\Psi_{i}\right|<x\right\}$ ), for $N_{\mathrm{FFT}}=128$, a Wiener phase noise with linewidth normalized to the symbol rate of either $1.2 \times 10^{-5}$ or $2 \times 10^{-4}$, and absence of CFO (namely, $\Delta F T_{c}=0$ ). In Fig. 6 the cdf of $\left|\Psi_{0}\right|$ is shown for the two considered linewidths.

As it can be seen, the slower the phase noise, the closer to 1 $\left|\Psi_{0}\right|$. On the other hand, for faster phase noise, the coefficient sometimes can have a magnitude much smaller than one. Similarly, in Fig. 7 the cdfs of coefficients $\left|\Psi_{1}\right|$ and $\left|\Psi_{2}\right|$ are shown for the two mentioned phase noise linewidth values. In this case, the coefficients are close to zero if the phase noise is slow, while their magnitude can be significant for faster phase noise.

Even if the phase noise is sufficiently slow so that $\left|\Psi_{0}\right| \simeq 1$ and $\left|\Psi_{i}\right| \simeq 0$, still the phase $\Psi_{0}$ introduces a rotation on all transmitted symbols which must be taken into account. However, we point out that, by using the detection strategy described in Section III.B, namely transmission of differentially encoded symbols and the adoption of the asynchronous detection algorithm in [20] at the receiver, the constant (across subcarriers)

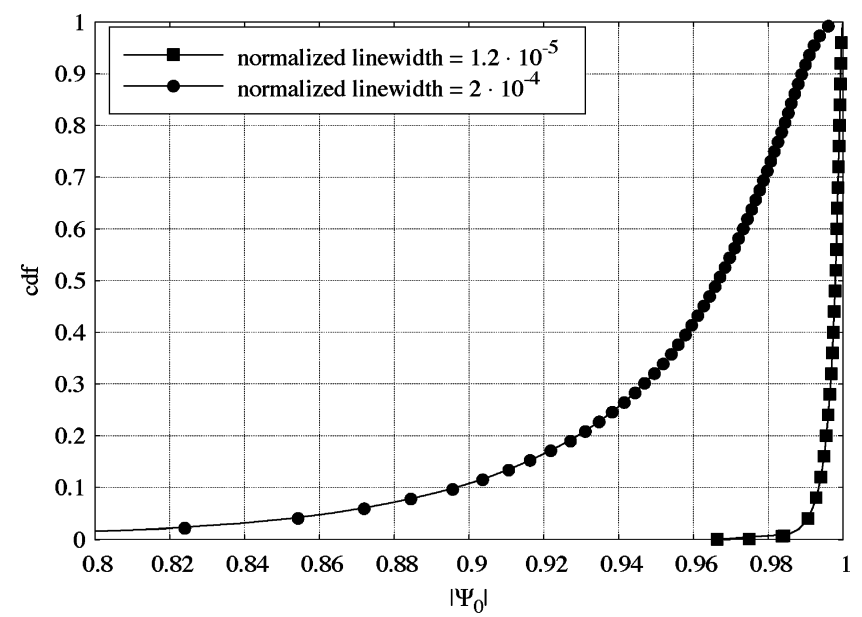

Fig. 6. Cdf of $\left|\Psi_{0}\right|$, for the two considered phase noise speeds.

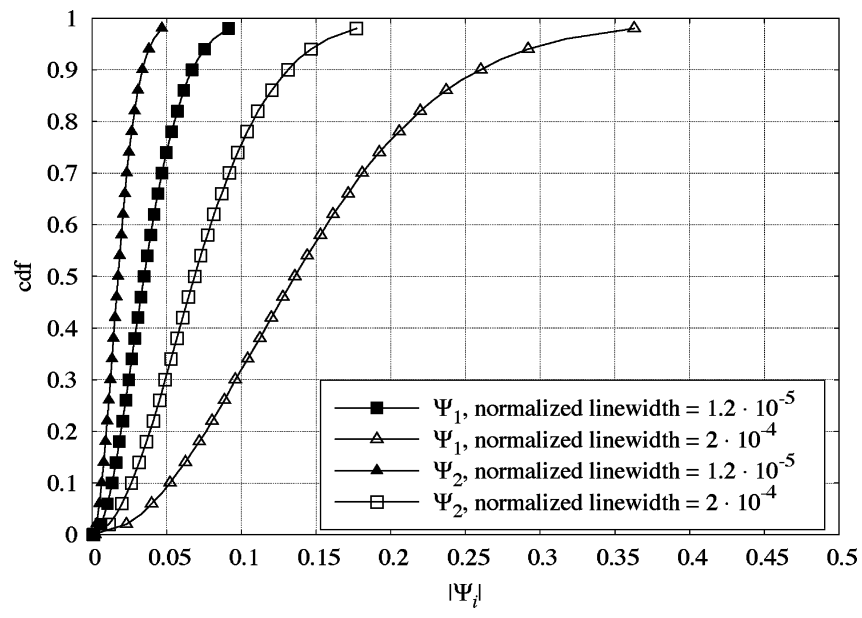

Fig. 7. Cdf of $\left|\Psi_{1}\right|$ and $\left|\Psi_{2}\right|$, for the two considered phase noise linewidth.

phase $\arg \left(\Psi_{0}\right)$ is automatically taken into account without further processing.

Countermeasures to CFO and phase noise in OFDM systems can be conceived either before or after the FFT block at the receiver (in the time or in the frequency domain). A compensation before FFT must be preferred since it allows (when perfect) to remove ICI. As an example, this can be accomplished by sacrificing some of the data subcarriers to transmit an unmodulated pilot tone that, properly filtered, provides the required reference for phase estimation and compensation [12]. In this case, the power allocated to the unmodulated subcarrier must be optimized. In fact, the higher its power, the more reliable the carrier estimate, but also the higher the energy not associated to data (and hence wasted). In the numerical results related to the performance of OFDM in the presence of phase noise (Section VII.B), we will extract the pilot tone with the best possible filter, i.e., a PLL whose equivalent bandwidth is optimized for the phase noise at hand.

Alternatively, one could work after the FFT block at the receiver. Compensation of phase noise and CFO in this case means ICI compensation through one of the algorithms proposed in the literature for such a purpose (e.g., see [48]-[51]). 
However, the computational complexity of these ICI compensation algorithms is definitely unaffordable in the considered context, due to the large signaling rates.

\section{NONLINEAR Distortions}

Another important source of impairment for high-speed optical transmissions are fiber nonlinear effects. The impact of nonlinearities is more relevant for increasing signalling rates, as it depends on the transmitted signal power. Basically, wavelength division multiplexing (WDM) communications suffer from distortions caused by the signal itself, like self-phase modulation (SPM), and by other adjacent channels, like cross-phase modulation (XPM) and four-wave mixing (FWM) [60]. As the impact of nonlinear effects increases with the transmitted power, a trade-off must be found between the penalty given by the ASE noise and the penalty given by nonlinear effects. The transmitted power can be raised if techniques to mitigate nonlinear effects are employed. Many works have been carried out in this field; it is worth noting that unlike other optical channel impairments which do not entail main differences from wireless or wired channels, this effect is typical of fiber-optic communications, and so are the derived mitigation techniques. Most of these solutions, moreover, can be applied to both OFDM and SC systems: optical conjugation [61], [62], backward propagation [63], nonlinear phase noise compensation [39]. Each technique has its own merits and drawbacks, but, since the scope of this work is a comparison between OFDM and SC systems, the attention will be focused on their different behavior in the nonlinear regime.

\section{A. Single-Carrier}

Nonlinear effects on SC modulations have been deeply investigated as a major source of impairment in WDM transmissions. Since, as shown, linear effects in coherent optical systems can be completely compensated for, nonlinear effects become one of the most severe limiting factors in long-haul optical systems. As mentioned above, we just focus the attention on the performance of the proposed receiver for SC modulations, disregarding compensation methods common to both SC and OFDM systems.

\section{B. $O F D M$}

A well known drawback of OFDM, which has been widely analyzed in the context of wireless communications, is its large PAR, as opposed to SC modulations [64]. In other words, for a given average transmitted power, the continuous-time OFDM signal has a much larger dynamic range than any single-carrier signal. While in a linear regime this characteristic does not affect system performance, in the presence of nonlinear fiber effects, which depend on the instantaneous power of the transmitted signal, a large performance degradation is unavoidable.

A trivial but usually ineffective way to overcome this problem is the clipping of the transmitted signal [65], so as to reduce its dynamic range. Besides the peak regrowth phenomenon which arises when clipping is carried out on the discrete-time signal, this approach has several severe drawbacks. First of all, the power spectra of the transmitted signal may dramatically change after clipping, an issue that in certain circumstances is unacceptable. For instance, in a multichannel communication system, a non-negligible energy could leak to neighbouring channels, thus causing interference. Furthermore, clipping the time-domain signal is equivalent to adding a further pseudo-random noise term to the frequency-domain received samples, in addition to the additive Gaussian noise. Although clip-noise estimation and compensation algorithms, to be deployed at the receiver, have been proposed in the literature (e.g., see [66]), their complexity is unaffordable for high-speed optical systems. Unfortunately, since in order to effectively reduce the impact of nonlinear effects a low enough clip level must be used, the resulting clip noise can completely destroy transmission.

Due to the above mentioned drawbacks of clipping, several more advanced techniques, aiming at the reduction of the PAR of an OFDM signal, have been proposed in the literature (see [16] for a survey). Despite the remarkable research efforts toward effective PAR reduction techniques, all solutions have at least one of the following quite serious drawbacks:

- very limited PAR reduction performance (e.g., 1-2 dB in practical scenarios);

- energy and spectral overhead due to the introduction of reserved subcarriers, that do not carry useful information, but are used for PAR reduction only;

- further noise at the receiver (as in the clipping approach);

- transmission of a side information, which is required at the receiver for correct detection of the transmitted OFDM symbol. Besides the overhead it introduces, this side information must be highly protected by means of powerful error-correcting codes, since even a single bit error for the side information entails the complete loss of the whole OFDM symbol;

- a very large computational complexity increase, usually at the transmitter side;

- remarkable distortion of the power spectra of the transmitted signals.

Due to the above drawbacks, to the best of our knowledge, the use of a PAR reduction technique when OFDM is employed in optical communication systems has been proposed only in [40] and the clipping approach seems to be the preferred way for reducing the impact of nonlinear effects, although we point out that a thorough performance analysis must be carried out in order to ensure that the performance degradation due to clip noise stays bounded. Finally, we would like to mention that OFDM shows a better resilience to nonlinear effects in dispersion-unmanaged links, where the performance gap with respect to SC almost disappears, since the presence of a significant amount of GVD has a useful impact on PAR. However, although a benefit can be seen in the fact that dispersion maps could be designed with more relaxed constraints, nowadays all installed links are designed as to minimize the residual dispersion and, moreover, a CP length as that needed in dispersion-unmanaged links would heavily affect the energy efficiency of the system.

\section{COMPLeXITY, EFFicienCy, AND TECHNOLOGICAL ISSUES}

We already showed that there are several theoretical reasons explaining why OFDM cannot exploit its potential advantages with respect to SC modulations in optical channels. In addition, some further straightforward considerations on computational complexity and spectral or energy efficiency can be 
drawn, which further support the thesis that no real benefits come from using OFDM in SMFs, at least in the $100 \mathrm{Gbps}$ scenario.

\section{A. Single-Carrier}

The main difficulties for $100 \mathrm{Gbps} \mathrm{SC}$ transmissions reside at the receiver, since at the transmit end a simple Mach-Zehnder modulator is sufficient, at least in the QPSK case. The received signal must be oversampled to obtain a sufficient statistics, allowing for an effective signal processing. In a $100 \mathrm{Gbps}$ polarization-multiplexed QPSK transmission, adopting the described parallel architecture to implement the two-dimensional equalizer in time or frequency domain, using, as an example, an oversampling factor of 1.2 samples per symbol and neglecting the unavoidable overhead due to forward error correction, only the first receiver block, i.e., the automatic frequency control loop, must process 30 Gsamples per second, whereas the remaining blocks can work at a much lower speed due to parallelization. As mentioned, the degree of parallelism is completely a degree of freedom and can be chosen according to the implementation requirements only. Regarding the ADC resolution, 6 bits are sufficient to represent each (real or imaginary) component of the received sample on each polarization [18]. A lower number of bits can be adopted at a price of a performance degradation.

\section{B. $O F D M$}

One of the main issues when dealing with optical OFDM is the availability of the required devices needed to effectively implement both transmitter and receiver [24]. High-resolution DACs at the transmitter and ADCs at the receiver are in general required, due to the fact that the transmitted OFDM signal usually exhibits a very high dynamic range for a given average power, and the quantization noise power must be kept below other noise sources. DACs with at least $7 \div 8$ bits at the transmitter [40] and ADCs with 8-bit resolution at the receiver [6] are claimed to be necessary to have no performance loss, at least in systems with inline dispersion compensation. ${ }^{8}$ Instead, the requirement on the speed of ADCs is the same as in SC transmissions, as using $20 \%$ of virtual subcarriers is equivalent to an oversampling factor of 1.2 samples per symbol. These converters are now becoming commercially available. The main technological problem turns out to be the speed of the required DACs at the transmitter. As a matter of fact, nowadays, implementing a clean OFDM modulation with a single IFFT/FFT pair turns out to be not feasible, due to the unavailability of DACs with the required speed and resolution. A $100 \mathrm{Gbps}$ OFDM transmission is rather implemented through some alternative solutions, like orthogonal band multiplexing (OBM) [33] or subcarrier multiplexing (SCM) [30]. Here, the overall data stream is split into several orthogonal sub-blocks for separate OFDM modulations, each centered at different wavelengths, with a proper choice of frequency offsets in order to exploit the orthogonality of subcarriers across different sub-blocks. These techniques clearly weigh on the cost and on the complexity of the system, both at the transmitter and at the receiver, depending on the choice of multiplexing the sub-bands directly

\footnotetext{
${ }^{8}$ In systems without inline dispersion compensation, the effect of PAR reduction due to GVD can reduce the requirements on the ADC resolution [11].
}

in the optical domain or using an intermediate frequency (IF) conversion stage, in the electrical domain [26].

Another aspect to be considered is that, due to the higher sensitivity to phase noise, expensive narrow-linewidth lasers must be employed. As opposed to SC, OFDM also exhibits several overheads which remarkably reduce the spectral efficiency and/or the energy efficiency of the system. As discussed in Section III.B, the CP wastes both energy and transmission time (bandwidth), since it consists in data transmitted twice. As shown, in order to avoid interference, the $\mathrm{CP}$ length cannot be less than the impulse response length. On the other hand, due to phase noise and CFO, the OFDM symbol duration must be kept small enough so as to reduce the performance degradation stemming from ICI. Therefore, the overhead due to CP cannot be easily reduced, unless a complete redesign of the system is considered.

Thanks to the adoption of differential encoding and asynchronous detection at the receiver, explicit channel tracking can be avoided and the channel can be estimated only once through pilot symbols, thus avoiding a further overhead. On the contrary, the presence of a pilot carrier, used for phase noise compensation before FFT at the receiver side [12], represents another remarkable source of overhead since the relevant power is wasted and some subcarriers must be turned off for its placement. In addition, a filter able to extract it must be carefully designed in order to avoid performance degradation.

\section{NUMERICAL RESULTS}

We simulated a system with 4th-order Gaussian filters both at the transmitter and at the receiver with a $3 \mathrm{~dB}$ bandwidth equal to $0.8 / T$. The SC system is designed according to [18]. In particular, we adopt the automatic frequency control loop there described and the asynchronous strategies for detection and for the adjustment of the equalizer coefficients, both using $N=8$ past decisions [18]. The equalizer is implemented in the frequency domain and works with 1.2 samples per symbol. This oversampling factor was found to be sufficient to assure no performance loss with respect to the $b 2 b$ system. For a fair comparison, the OFDM system has $20 \%$ of virtual subcarriers and adopts the same asynchronous strategy after the two-dimensional one-tap equalizer. As mentioned, this is advantageous both for estimating the channel frequency response and for increasing the robustness to phase noise (although it cannot compensate for the arising ICI).

\section{A. Time-Invariant Linear Distortions}

We consider the performance in terms of the $E_{b} / N_{0}$ value required to obtain a BER of $10^{-4}, E_{b}$ being the received signal energy per information bit. In Figs. 8 and 9, performance is plotted versus the number $L_{c}$ of equalizer taps for the SC transmission, and versus $N_{\mathrm{CP}}$ for the OFDM system in correspondence of different values of $N_{\mathrm{FFT}}$. Two different links at 100 Gbps were taken into account. In the case of Fig. 8, a link with dispersion compensation having a residual dispersion $D_{r}=650 \mathrm{ps} / \mathrm{nm}$ and first order PMD, characterized by a power splitting ratio between the principal states of polarization of $\rho=0.5$ and differential group delay $\tau=20 \mathrm{ps}$, is considered. The second link is without in-line dispersion compensation 


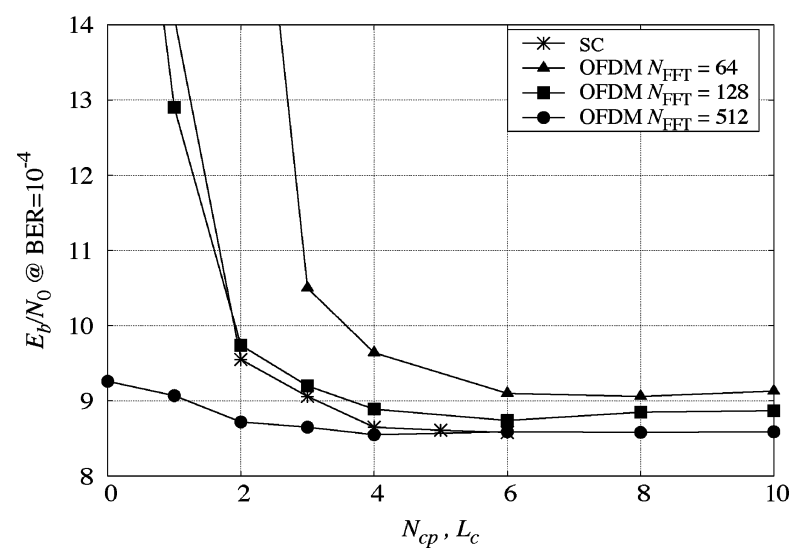

Fig. 8. Required $E_{b} / N_{0}$ to obtain a BER equal to $10^{-4}$ for different values of $N_{\mathrm{CP}}$ (OFDM case) and $L_{c}$ (SC case), in the presence of GVD and PMD (link with dispersion compensation).

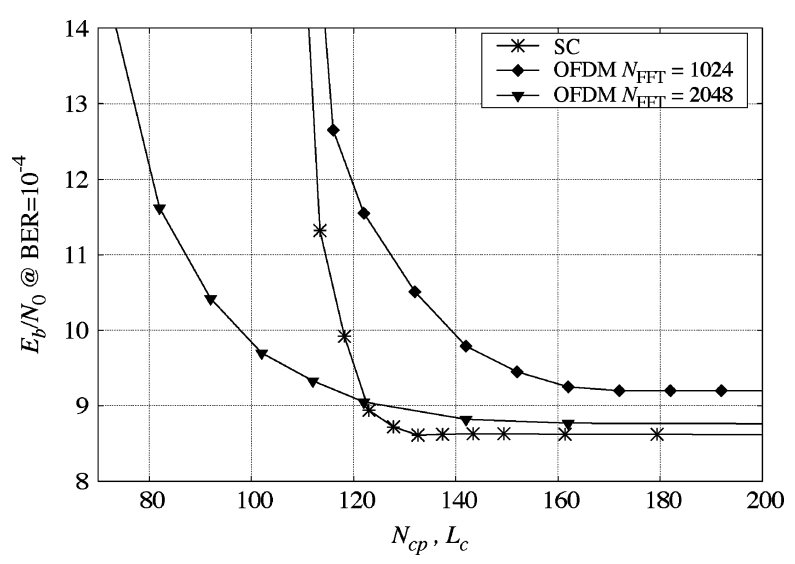

Fig. 9. Required $E_{b} / N_{0}$ to obtain a BER equal to $10^{-4}$ for different values of $N_{\text {CP }}$ (OFDM case) and $L_{c}$ (SC case), in the presence of GVD and PMD (link without dispersion compensation).

with $D_{r}=20000 \mathrm{ps} / \mathrm{nm}$ (corresponding to $1200 \mathrm{~km}$ of standard fiber), $\rho=0.5$, and $\tau=80 \mathrm{ps}$ and the corresponding results are reported in Fig. 9. In all cases, the performance improves when $L_{c}$ and $N_{\mathrm{CP}}$ increase and reaches the optimal value, corresponding to a $\mathrm{b} 2 \mathrm{~b}$ transmission (excepting, in the OFDM case, the energy loss due to the CP insertion), when $L_{c}=N_{\mathrm{CP}}+1=L$. For OFDM transmissions, an increase in the number of subcarriers has also the beneficial effect of a reduced ICI when the CP length is not sufficiently large. For the link without in-line dispersion compensation we considered larger values for $N_{\mathrm{FFT}}$ since otherwise the overhead due to the $\mathrm{CP}$ would be significant.

\section{B. Phase Noise and $C F O$}

In Fig. 10 we consider, for the system with dispersion compensation mentioned above, the sensitivity of SC and OFDM to an uncompensated CFO. In other words, the AFC of the SC scheme is turned off and no pilot tones are transmitted in the OFDM system to perform frequency estimation. No phase noise has been added, i.e., a very narrow laser linewidth has been considered. Moreover, nonlinear effects are absent. Different values of $N_{\text {FFT }}$ are considered all with $N_{\mathrm{CP}}=16$. The value of

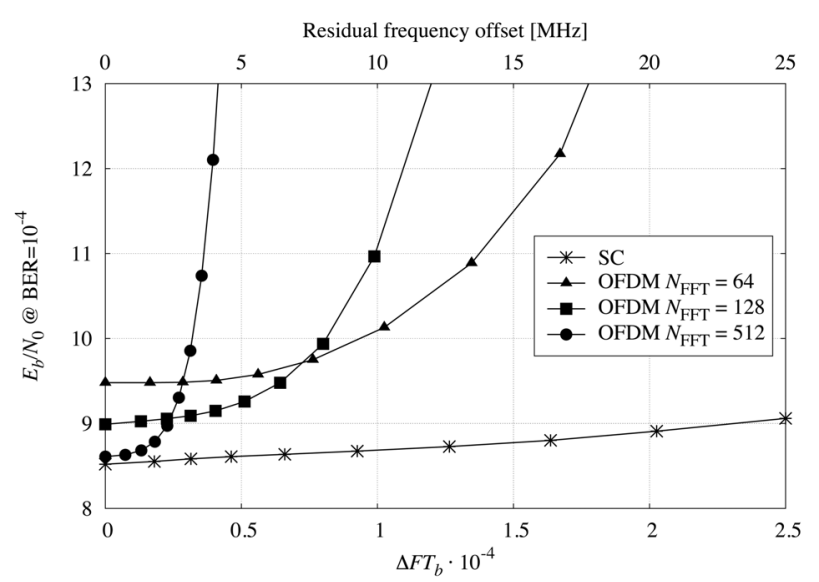

Fig. 10. Performance degradation in the presence of a constant CFO.

$E_{b} / N_{0}$ required to obtain a BER of $10^{-4}$ is shown as a function of the uncompensated CFO $\Delta F$ (values normalized to the symbol rate $1 / T$ in the lower scale and absolute values with reference to a QPSK-based polarization-multiplexed $100 \mathrm{Gbps}$ transmission in the upper scale). This figure allows to appreciate the remarkable performance degradation due to the uncompensated CFO, for a few different values of $N_{\mathrm{FFT}}$. In particular, the longer the OFDM symbols, the more sensitive the OFDM system to the uncompensated CFO. On the contrary, the SC scheme exhibits a larger robustness and thus lower-accuracy algorithms for carrier frequency estimation and compensation can be employed.

In Figs. 11 and 12 we consider the effect of the receiver phase noise, under the assumption of ideal CFO compensation, for the above mentioned compensated and uncompensated links. Transmit phase noise is neglected since it has usually a lower impact on the performance. Different OFDM symbol durations $\left(N_{\mathrm{FFT}}\right)$ have been considered. The CP length is $N_{\mathrm{CP}}=12$ for the compensated link and $N_{\mathrm{CP}}=162$ for the uncompensated link. The overhead due to $N_{\mathrm{CP}}$ was accounted for in the SNR computation. For the SC system $L_{c}=9$ for the compensated link, whereas $L_{c}=160$ for the uncompensated link. The $E_{b} / N_{0}$ required to obtain a BER of $10^{-4}$ is shown as a function of phase noise linewidth (normalized values in the lower scale, absolute values referred to a $100 \mathrm{Gbps}$ transmission in the upper scale). The above mentioned asynchronous SbS detection strategy is employed in the SC case, whereas for OFDM we considered two cases. The first one is based on the compensation in the time domain (before FFT at the receiver) and requires the transmission of a pilot tone (curves labeled with "PT") with optimized power. To insert the pilot tone, we turned off $3 \%$ of the overall subcarriers and, as in [12], we inserted the pilot tone in place of the subcarrier with $k=0$ in (3). As mentioned, the pilot is extracted by means of a PLL of optimized equivalent bandwidth. The second approach works after FFT and is based on the adoption of the above mentioned asynchronous detection strategy after the two-dimensional one-tap equalizer. This approach is therefore not able to compensate for ICI and hence has a worse performance. It can be seen that the robustness of OFDM depends on the symbol duration. In particular, the phase noise (but also the CFO) drives the system design 
TABLE I

SMF AND DCF LENGTHS FOR EACH SPAN IN THE SiMUlated OPTICAL LiNK

\begin{tabular}{|c|c|c|c|c|c|c|c|c|c|c|c|c|c|c|c|}
\hline SM & 96.3 & 90.8 & 90.6 & 100.2 & 96.9 & 83.6 & 94.5 & 86.3 & 88.9 & 91.9 & 60.8 & 80.5 & 91.8 & 94.1 & 0 \\
\hline$\overline{\mathrm{DCF}}(\mathrm{km})$ & 21.94 & $\overline{0}$ & $\overline{00}$ & 21.94 & 21.94 & $\overline{0}$ & 21.94 & 21.94 & $\overline{0}$ & $\overline{0}$ & 21.94 & 21.94 & 0 & 21.94 & 5.85 \\
\hline
\end{tabular}

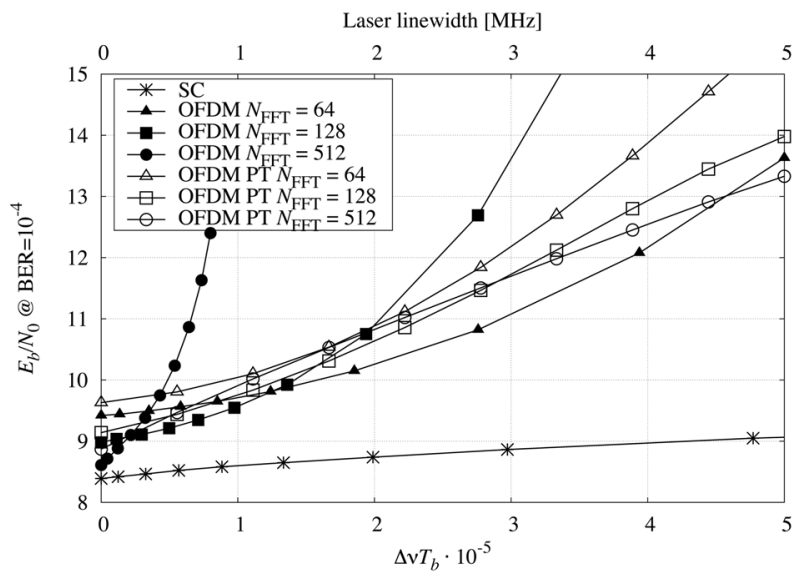

Fig. 11. Performance degradation in the presence of phase noise (link with dispersion compensation).

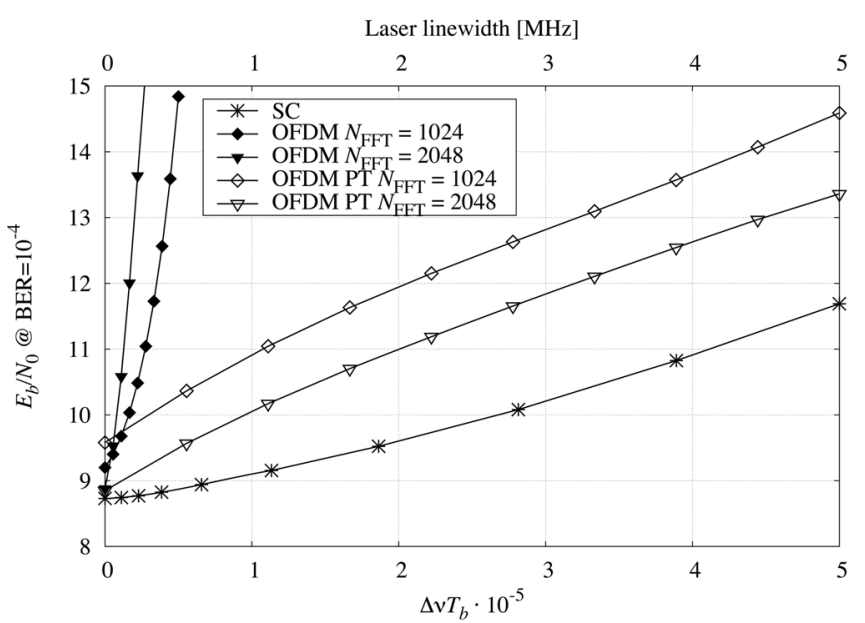

Fig. 12. Performance degradation in the presence of phase noise (link without dispersion compensation).

towards short symbols lengths, at least when the pilot tone is absent. Regarding the SC scheme, for both links the robustness is higher than that observed in OFDM.

\section{Nonlinear Distortions}

We now compare SC and OFDM over an existing dispersion-compensated WDM link of about $1200 \mathrm{~km}$, taking into account nonlinear effects. The link is composed of 14 spans of different length of standard SMF (dispersion of $16.46 \mathrm{ps} / \mathrm{nm} / \mathrm{km}$ ) with, at the end of each span, a dispersion compensating fiber (DCF) (dispersion $-113.7 \mathrm{ps} / \mathrm{nm} / \mathrm{km}$ ) and an inline single or dual stage optical amplifier (as reported in Table I). A fifteenth span made up by just a postcompensation DCF is present. The dispersion map is designed in order to leave a residual dispersion of about $D_{r}=100 \mathrm{ps} / \mathrm{nm}$. In Fig. 13, we considered a SC

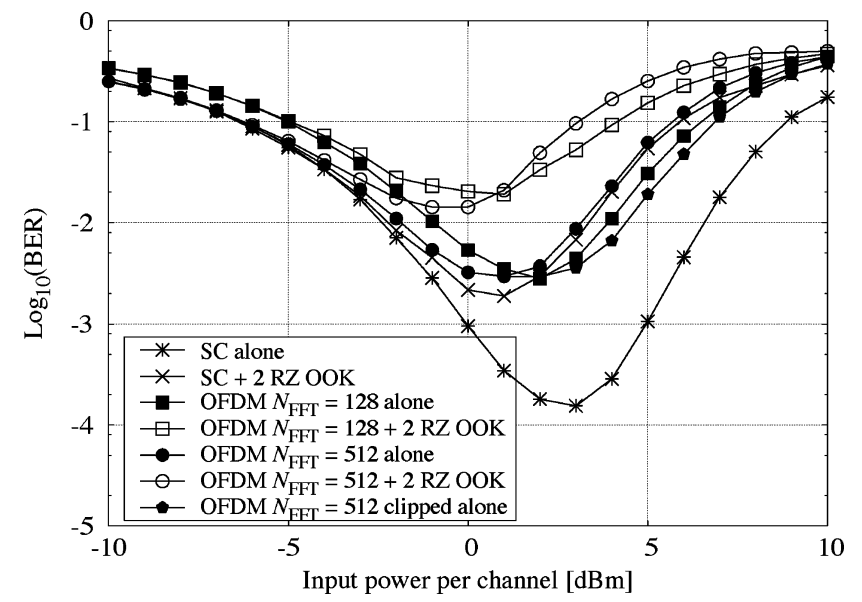

Fig. 13. BER versus input power per channel of OFDM and SC systems in a WDM scenario. The interfering channels are standard $10 \mathrm{Gbps}$ on-off keying (OOK) with $50 \mathrm{GHz}$ spacing. Link length equal to $1200 \mathrm{Km}$, residual dispersion equal to $100 \mathrm{ps} / \mathrm{nm}$.

system using a two-dimensional equalizer with $L_{c}=9$ and an OFDM system with $N_{\mathrm{FFT}}=128$ or 512 and $N_{\mathrm{CP}}=16$. The transmitted OFDM signal with $N_{\mathrm{FFT}}=512$ was also simulated using clipping at the trasmitter as proposed in [40], with an optimized clipping ratio equal to 1.5 (this parameter represents the ratio between the maximum allowed peak value and the signal root mean square value). The case of absence of adjacent channels is considered along with the case of a presence of two adjacent $10 \mathrm{Gbps}$ OOK channels. As expected, OFDM exhibits a worse performance (see also [17]).

For the same link, but in the absence of DCFs, the performance is shown in Fig. 14 in both cases of absence or presence of adjacent $10 \mathrm{Gbps}$ OOK channels, but in this figure $L_{c}=160$ for the SC system whereas $N_{\mathrm{FFT}}=2048$ and $N_{\mathrm{CP}}=162$ for the OFDM system. For the link with DCFs we also evaluated the effects of two adjacent $100 \mathrm{Gbps}$ SC or OFDM systems on a $10 \mathrm{Gbps}$ OOK channel. The relevant performance is shown in Fig. 15. In all figures, when present, interfering channels are always launched at the same power of the observed channel and the performance is shown as a function of the input power per channel. In the considered link, several filters, multiplexers and demultiplexers are envisaged, typically modeled as first- or fourth-order Gaussian optical filters with $3 \mathrm{~dB}$ bandwidths in the range $0.7 / T$ to $0.95 / T$.

In the presence of inline compensation, (Fig. 13) there is, as expected, a large advantage of SC over OFDM. However this advantage is reduced when side channels are present, since the effect of XPM prevails over SPM. In the case of absence of inline compensation (Fig. 14), OFDM and SC provide almost the same performance, both in presence and in absence of interferers. This is due to the beneficial spreading effect of GVD which reduces the signal PAR. Finally, from Fig. 15 it is 


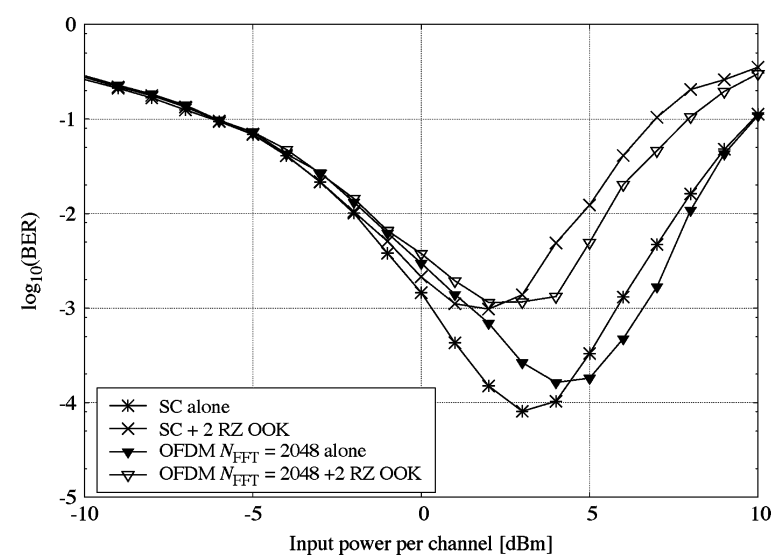

Fig. 14. BER versus input power per channel of OFDM and SC systems in an uncompensated WDM scenario. The interfering channels are standard $10 \mathrm{Gbps}$ on-off keying (OOK) with $50 \mathrm{GHz}$ spacing. Link length equal to $1200 \mathrm{Km}$, residual dispersion equal to $20000 \mathrm{ps} / \mathrm{nm}$.

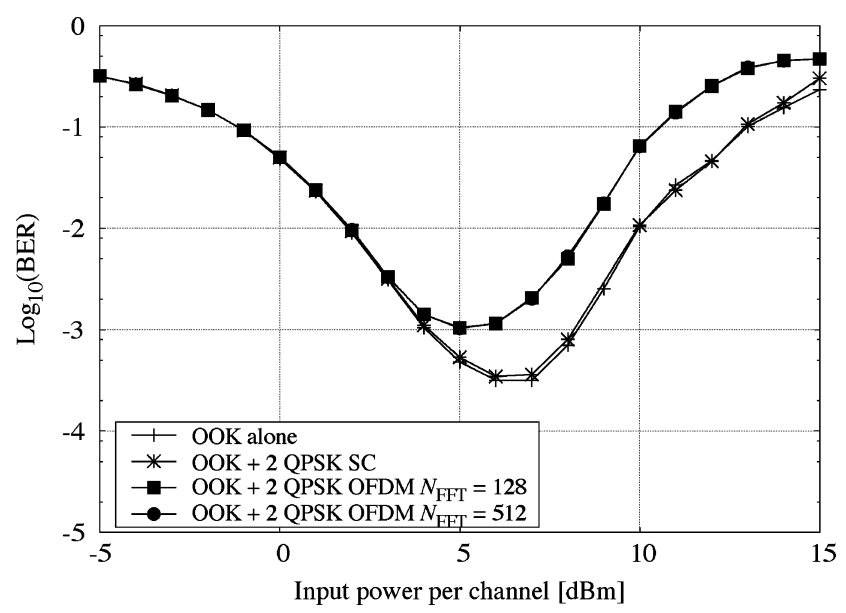

Fig. 15. BER versus input power per channel of an existing OOK systems in a WDM scenario. The interfering channels are $100 \mathrm{Gbps}$ OFDM and SC QPSK with $50 \mathrm{GHz}$ spacing. Link length equal to $1200 \mathrm{Km}$, residual dispersion equal to $100 \mathrm{ps} / \mathrm{nm}$.

clear that the OFDM system has a more detrimental effect on a 10 Gbps OOK channel.

\section{CONCLUSION}

The main advantage of OFDM, which made it a winning technology for a large number of communication standards like DVB, DSL, WiMax, is the possibility to match the transmitted signal spectrum to the particular channel characteristics, like non-flat amplitude responses, due to fading, or multipath, etc. The effective exploitation of the channel is also possible through several techniques which improve OFDM efficiency, like bit and power loading, pulseshaping, channel shortening. All these peculiar features of OFDM have a lower impact in optical communication, as the channel amplitude response is almost flat (neglecting minor impairments like polarization dependent losses and imperfections in the frequency responses of electrical and optical filters), and the high data rates prevent taking advantage from sophisticated signal processing.
The main reasons that drive the interest in OFDM are the simple compensation of linear impairments and the implicit parallelization given by the IFFT/FFT operations, which lowers the processing speed at the receiver. As demonstrated, a proper design of a SC transmission system can provide the same benefits without incurring in the tight constraints of OFDM in terms of frequency and phase errors, in its penalty due to nonlinear effects, in its lack of spectral and energy efficiency, in its difficult implementation due to technological limits.

A possible advantage of OFDM could be its scalability with higher bit rates, but, as shown, it is almost impossible nowadays to implement a convenient OFDM system even for 100 Gbps. As a conclusion, there are nowadays significant technological limits to a cheap and convenient implementation of OFDM, whereas SC modulations take advantage of a consolidated know-how and technology, relegating the efforts for realtime implementations only to the progress in the speed of optical and electronic devices.

\section{REFERENCES}

[1] G. Raybon and P. J. Winzer, " $100 \mathrm{~Gb} / \mathrm{s}$ challenges and solutions," in Proc. Optical Fiber Commun. Conf. (OFC'08), San Diego, CA, USA, Feb. 2008, paper OTuG1.

[2] M. Duelk, "Next generation $100 \mathrm{G}$ Ethernet," in Proc. Eur. Conf. Opt. Comm., Glasgow, U.K., Sep. 2005, paper Tu 3.1.2.

[3] J. A. C. Bingham, "Multicarrier modulation for data transmission: An idea whose time has come," IEEE Commun. Mag., pp. 5-14, May 1990.

[4] B. L. Floch, M. Alard, and C. Berrou, "Coded orthogonal frequency division multiplex," Proc. IEEE, vol. 83, pp. 982-996, Jun. 1995.

[5] Z. Wang and G. B. Giannakis, "Wireless multicarrier communications," IEEE Signal Processing Mag., vol. 17, pp. 29-48, May 2000.

[6] J. A. C. Bingham, ADSL, VDSL, and Multicarrier Modulation. New York: John Wiley \& Sons, 2000.

[7] C. Y. Wong, R. S. Cheng, K. B. Letaief, and R. D. Murch, "Multiuser OFDM with adaptive subcarrier, bit, and power allocation," IEEE $J$. Select. Areas Commun., vol. 17, pp. 1747-1758, Oct. 1999.

[8] J. Jang and K. B. Lee, "Transmit power adaptation for multiuser OFDM systems," IEEE J. Select. Areas Commun., vol. 21, pp. 171-178, Feb. 2003.

[9] B. J. C. Schmidt, A. J. Lowery, and J. Armstrong, "Experimental demonstrations of electronic dispersion compensation for long-haul transmission using direct-detection optical OFDM," J. Lightwave Tech., vol. 26, pp. 196-203, Jan. 2008.

[10] W. Shieh, H. Bao, and Y. Tang, "Coherent optical OFDM: Theory and design," Opt. Express, vol. 16, pp. 841-859, Jan. 2008.

[11] S. L. Jansen, B. Spinnler, I. Morita, S. Randel, and H. Tanaka, "100 GbE: QPSK versus OFDM," Optical Fiber Technology, vol. 15, pp. 407-413, Oct.-Dec. 2009.

[12] S. L. Jansen, I. Morita, T. C. W. Schenk, N. Takeda, and H. Tanaka, "Coherent optical 25.8-Gb/s OFDM transmission over 4160-km SSMF," J. Lightwave Tech., vol. 26, pp. 6-15, Jan. 2008.

[13] L. Tomba, "On the effect of Wiener phase noise in OFDM systems," IEEE Trans. Commun., vol. 46, pp. 580-583, May 1998.

[14] W. Kozek and A. F. Molisch, "Nonorthogonal pulseshapes for multicarrier communications in doubly dispersive channels," IEEE J. Select. Areas Commun., vol. 16, pp. 1579-1589, Oct. 1998.

[15] T. Strohmer and S. Beaver, "Optimal OFDM design for time-frequency dispersive channels," IEEE Trans. Commun., vol. 51, pp. 1111-1122, Jul. 2003.

[16] S. H. Han and J. H. Lee, "An overview of peak-to-average power ratio reduction techniques for multicarrier transmission," IEEE Wireless Commun., vol. 12, pp. 56-65, Apr. 2005.

[17] X. Liu, F. Buchali, R. W. Tkach, and S. Chandrasekhar, "Mitigation of fiber nonlinear impairments in polarization division multiplexed OFDM transmission," Bell Labs Tech. J., vol. 14, pp. 47-59, 2010.

[18] G. Colavolpe, T. Foggi, E. Forestieri, and G. Prati, "Robust multilevel coherent optical systems with linear processing at the receiver," J. Lightwave Tech., vol. 27, pp. 2357-2369, Jul. 1, 2009.

[19] L. G. Kazowsky, S. Benedetto, and A. Willner, Optical Fiber Cоттиnication Systems. Norwood, MA: Artec House, 1996. 
[20] G. Colavolpe and R. Raheli, "Noncoherent sequence detection," IEEE Trans. Commun., vol. 47, pp. 1376-1385, Sep. 1999.

[21] A. V. Oppenheim and R. W. Schafer, Discrete-Time Signal Processing, 2nd ed. , New Jersey: Prentice Hall, 1999.

[22] J. Armstrong, "OFDM for optical communications," J. Lightwave Tech., vol. 27, pp. 189-204, Feb. 2009.

[23] A. J. Lowery and J. Armstrong, "Orthogonal-frequency-division-multiplexing for dispersion compensation of long-haul optical systems," Opt. Express, vol. 14, pp. 2079-2084, Mar. 2006.

[24] S. L. Jansen, I. Morita, K. Forozesh, S. Randel, D. van den Borne, and H. Tanaka, "Optical OFDM a hype or is it for real?," in Proc. European Conf. on Optical Commun. (ECOC'08), Brussels, Belgium, Sep. 2008, Paper Mo.3.E.3.

[25] W. Shieh, X. Yi, Y. Ma, and Y. Tang, "Theoretical and experimental study on PMD-supported transmission using polarization diversity in coherent optical OFDM systems," Opt. Express, vol. 15, pp. 9936-9947, Aug. 2007.

[26] S. L. Jansen, I. Morita, T. C. W. Schenk, and H. Tanaka, "121.9-Gb/s PDM-OFDM transmission with $2-\mathrm{b} / \mathrm{s} / \mathrm{Hz}$ spectral efficiency over 1000-km of SSMF," J. Lightwave Tech., vol. 27, pp. 177-188, Feb. 2009.

[27] S. B. Weinstein and P. M. Ebert, "Data transmission by frequency-division multiplexing using the discrete Fourier transform," IEEE Trans. Commun., vol. com-19, pp. 628-634, Oct. 1971.

[28] W. Rhee and J. M. Cioffi, "Increase in capacity of multiuser OFDM system using dynamic subchannel allocation," in Proc. Vehicular Tech. Conf., Tokio, Japan, 2000, pp. 1085-1089.

[29] W. Shieh, Q. Yang, and Y. Ma, "107 Gb/s coherent optical OFDM transmission over 1000-km SSMF fiber using orthogonal band multiplexing," Opt. Express, vol. 16, pp. 6378-6386, Apr. 2008.

[30] T. Kobayashi, E. Yamazaki, E. Yamada, H. Masuda, A. Sano, E. Yoshida, Y. Miyamoto, K. Ishihara, Y. Kudo, R. Takatori, and M. Mizoguchi, "Ultra long-haul transmission over $6000 \mathrm{~km}$ of $100 \mathrm{~Gb} / \mathrm{s}$ serial signal by using coherent detection," in Proc. Optical Fiber Commun. Conf. (OFC'09), San Diego, CA, USA, Mar. 2009, paper OWW4.

[31] X. Liu and F. Buchali, "A novel channel estimation method for PDMOFDM enabling improved tolerance to WDM nonlinearity," in Proc. Optical Fiber Commun. Conf. (OFC'09), San Diego, CA, USA, Mar. 2009, paper OWW5.

[32] W. Shieh, X. Yi, and Q. Yang, "Coherent optical OFDM: Has its time come?," J. Opt. Networking, vol. 7, pp. 234-255, Mar. 2008.

[33] Q. Yang, Y. Tang, and W. Shieh, "Experimental demonstration and numerical simulation of $107-\mathrm{Gb} / \mathrm{s}$ high spectral efficiency coherent optical OFDM,” J. Lightwave Tech., vol. 27, pp. 168-176, Feb. 2009.

[34] X. Yi, W. Shieh, and Y. Ma, "Phase noise effects on high spectral efficiency coherent optical OFDM transmission," J. Lightwave Tech., vol. 26, pp. 1309-1316, May 2008.

[35] X. Yi, W. Shieh, and Y. Tang, "Phase estimation for coherent optical OFDM," IEEE Photon. Technol. Lett., vol. 19, pp. 919-921, Jun. 2007.

[36] W. Shieh, "Maximum-likelihood phase and channel estimation for coherent optical \{OFDM\}," IEEE Photon. Technol. Lett., vol. 20, pp. 605-607, Apr. 2008.

[37] Y. Tang, Y. Ma, and W. Shieh, "107 Gb/s CO-OFDM transmission with inline chromatic dispersion compensation," in Proc. Optical Fiber Commun. Conf. (OFC'09), San Diego, CA, USA, Mar. 2009, paper OWW3.

[38] A. J. Lowery, "Fiber nonlinearity pre- and post-compensation for long-haul optical links using OFDM," Opt. Express, vol. 15, pp. 12965-12970, Sep. 2007.

[39] K.-P. Ho and J. M. Kahn, "Electronic compensation technique to mitigate nonlinear phase noise," J. Lightwave Tech., vol. 22, pp. 779-783, Mar. 2004.

[40] D. J. F. Barros and J. M. Kahn, "Optical modulator optimization for orthogonal frequency-division multiplexing," J. Lightwave Tech., vol. 27, pp. 2370-2378, Jul. 1, 2009.

[41] Y. Tang, K.-P. Ho, and W. Shieh, "Coherent optical OFDM transmitter design employing predistortion," IEEE Photon. Technol. Lett., vol. 20, pp. 954-956, Jun. 2008.

[42] X. Liu, F. Buchali, and R. W. Tkach, "Improving the nonlinear tolerance of polarization-division-multiplexed CO-OFDM in long-haul fiber transmission," J. Lightwave Tech., vol. 27, pp. 3632-3640, Aug. 2009.

[43] E. Forestieri and L. Vincetti, "Exact evaluation of the Jones matrix of a fiber in the presence of polarization mode dispersion of any order," $J$. Lightwave Technol., vol. 17, pp. 1898-1909, Dec. 2001.
[44] H. Bulow, W. Baumert, H. Schmuck, F. Mohr, T. Schulz, F. Kuppers, and W. Weiershausen, "Measurement of the maximum speed of pmd fluctuation in installed field fiber," in Proc. Optical Fiber Commun. Conf. (OFC'99), Feb. 1999, Paper We4-1.

[45] R. Kudo, T. Kobayashi, K. Ishihara, Y. Takatori, A. Sano, E. Yamada, H. Masuda, Y. Miyamoto, and M. M. , "Two-stage overlap frequency domain equalization for long-haul optical systems," in Proc. Optical Fiber Commun. Conf. (OFC'09), San Diego, CA, USA, Mar. 2009, paper OMT3.

[46] M. Kuschnerov, F. N. Hauske, K. Piyawanno, B. Spinnler, M. S. Alfiad, A. Napoli, and B. Lankl, "DSP for coherent single-carrier receivers," J. Lightwave Tech., vol. 27, pp. 3614-3622, Aug. 2009.

[47] Q. Yang, W. Shieh, and Y. Ma, "Bit and power loading for coherent optical OFDM," IEEE Photon. Technol. Lett., vol. 20, pp. 1305-1307, Aug. 2008.

[48] W. J. Jeon, K. H. Chang, and Y. S. Cho, "An equalization technique for orthogonal frequency-division multiplexing systems in time-variant multipath channels," IEEE Trans. Commun., vol. 47, pp. 27-32, Jan. 1999.

[49] Y. Choi, P. J. Voltz, and F. A. Cassara, "On channel estimation and detection for multicarrier signals in fast and selective Rayleigh fading channels," IEEE Trans. Commun., vol. 49, pp. 1375-1387, Aug. 2001

[50] X. Cai and G. B. Giannakis, "Bounding performance and suppressing intercarrier interference in wireless mobile OFDM," IEEE Trans. Commun., vol. 51, pp. 2047-2056, Dec. 2003.

[51] P. Schniter, "Low-complexity equalization of OFDM in doubly selective channels," IEEE Trans. Signal Processing, vol. 52, pp. 1002-1011, Apr. 2004.

[52] P. J. W. Melsa, R. C. Younce, and C. E. Rohrs, "Impulse response shortening for discrete multitone transceivers," IEEE Trans. Commun., vol. 44, pp. 1662-1672, Dec. 1996.

[53] A. Tkacenko and P. P. Vaidyanathan, "A low-complexity eigenfilter design method for channel shortening equalizers for DMT systems," IEEE Trans. Commun., vol. 51, pp. 1069-1072, Jun. 2003.

[54] E. Ip and J. M. Kahn, "Feedforward carrier recovery for coherent optical communications," J. Lightwave Tech., vol. 25, pp. 2675-2692, Sep. 2007.

[55] M. Russell and G. L. Stüber, "Interchannel interference analysis of OFDM in a mobile environment," in Proc. Vehicular Tech. Conf., 1995, vol. 2, pp. 820-824.

[56] Y. Li and J. L. J. Cimini, "Bounds on the interchannel interference of OFDM in time-varying impairments," IEEE Trans. Commun., vol. 49, pp. 401-404, Mar. 2001.

[57] A. Stamoulis, S. N. Diggavi, and N. Al-Dhahir, "Intercarrier interference in MIMO OFDM," IEEE Trans. Signal Processing, vol. 50, pp. 2451-2464, Oct. 2002

[58] T. Pollet, M. V. Bladel, and M. Moeneclaey, "BER sensitivity of OFDM systems to carrier frequency offset and Wiener phase noise," IEEE Trans. Commun., vol. 43, pp. 191-193, Feb. 1995.

[59] K. Nikitopoulos and A. Polydoros, "Phase-impairment effects and compensation algorithms for OFDM systems," IEEE Trans. Commun., vol. 53, pp. 698-707, Apr. 2005

[60] G. P. Agrawal, Nonlinear Fiber Optics, 3rd ed. San Diego, CA: Academic Press, 2001.

[61] S. Watanabe and M. Shirasaki, "Exact compensation for both chromatic dispersion and kerr effect in a transmission fiber using optical phase conjugation," J. Lightwave Tech., vol. 14, pp. 243-248, Mar. 1996.

[62] C. Lorattanasane and K. Kikuchi, "Design theory of long-distance optical transmission systems using midway optical phase conjugation," $J$. Lightwave Tech., vol. 15, pp. 948-955, Jun. 1997.

[63] E. Ip and J. M. Kahn, "Compensation of dispersion and nonlinear impairments using digital backpropagation," J. Lightwave Tech., vol. 26, pp. 3416-3425, Oct. 2008.

[64] J. Tellado, "Peak to Average Power Reduction for Multicarrier Modulation," Ph.D. Dissertation proposal, Stanford University, Palo Alto, CA, 2000, tech. rep.

[65] X. Li and L. J. Cimini, "Effects of clipping and filtering on the performance of OFDM," in Proc. Vehicular Tech. Conf., Phoenix, AZ, May 1997, pp. 1634-1638.

[66] H. Chen and A. M. Haimovich, "Iterative estimation and cancellation of clipping noise for OFDM signals," IEEE Commun. Letters, vol. 7, pp. 305-307, Jul. 2003. 


\begin{abstract}
Alan Barbieri received the Dr.Ing. degree in telecommunication engineering (cum laude) and the Ph.D. degree in information technology from the University of Parma, Parma, Italy, in 2003 and 2007, respectively.

Since July 2009 he is with Qualcomm Corporate R\&D Center, Qualcomm Inc., San Diego, CA. Previously he was associate professor at the Scuola Superiore Sant'Anna, Pisa, Italy, after holding a post-doc position at the Dept. of Information Technology, University of Parma. In 2007 he was a visiting scholar at the Ming Hsieh Department of Electrical Engineering, University of Southern California, Los Angeles, CA. During summer 2008 he was a visiting faculty at the Qualcomm Corporate R\&D Center, Qualcomm Inc., San Diego, CA. His main research interests include digital transmission theory and information theory, with particular emphasis on channel coding, iterative joint detection and decoding algorithms, estimation of unknown parameters and algorithms for synchronization. He participates in several research projects funded by the European Space Agency (ESA-ESTEC) and important telecommunication companies.
\end{abstract}

Giulio Colavolpe was born in Cosenza, Italy, in 1969. He received the Dr. Ing. degree in telecommunications engineering (cum laude) from the University of Pisa, Italy, in 1994 and the Ph.D. degree in information technologies from the University of Parma, Italy, in 1998.

Since 1997, he has been at the University of Parma, Italy, where he is now an Associate Professor of Telecommunications. In 2000, he was Visiting Scientist at the Institut Eurécom, Valbonne, France. His main research interests include digital transmission theory, adaptive signal processing, channel coding and information theory. His research activity has led to several scientific publications in leading international journals and conference proceedings and a few industrial patents. He is also coauthor of the book Detection Algorithms for Wireless Communications, with Applications to Wired and Storage Systems (New York: John Wiley \& Sons, 2004). He is currently serving as an Editor for the IEEE TRANSACTIONS ON WIRELESS COMMUNICATIONS and as an Executive Editor for the European Transactions on Telecommunications. He received the best paper award at the 13th International Conference on Software, Telecommunications and Computer Networks (SoftCOM'05), Split, Croatia, September 2005 and the best paper award for Optical Networks and Systems at the IEEE International Conference on Communications (ICC 2008), Beijing, China, May 2008. He is also the principal investigator of several research projects funded by the European Space Agency (ESA-ESTEC) and important telecommunications companies.

Tommaso Foggi was born in Parma, Italy, on May 7, 1978. He received the Dr. Ing. degree in telecommunications Engineering and the Ph.D. in information technology from the University of Parma, Parma, Italy, in 2003 and 2008, respectively.

From July 2003 to July 2004 he was granted a CNIT scholarship at Photonic Networks National Laboratory, Pisa, and another CNIT scholarship from July 2005 to July 2006 at Dipartimento di Ingegneria dell'Informazione (DII), Università Degli Studi di Parma, Parma. Since June 2009 he is now a research fellow of National Inter-university Consortium for Telecommunications (CNIT), after holding post-doc collaboration contracts with University of Parma, CNIT and Scuola Superiore Sant'Anna in 2008 and 2009.

His main research interests include digital signal processing for optical communications, i.e., equalization, estimation and compensation techniques, advanced modulation formats, detection algorithms and receiver design. His activity also contributed to several research projects funded by the Italian Ministry of Education, University and Research (MIUR) or by International companies.
Enrico Forestieri (S'91-M'92) was born in Milazzo, Italy, in 1960. He received the Dr. Ing. degree in electronics engineering from the University of Pisa, Pisa, Italy, in 1988. From 1989 to 1991 he has been a postdoctoral scholar at the University of Parma, Italy, working on optical communication systems. From 1991 to 2000 he was a Research Scientist and faculty member of the University of Parma. Since 2001 he is with Scuola Superiore Sant'Anna di Studi Universitari e di Perfezionamento, Pisa, Italy, where currently he is Professor of Telecommunications. His research interests are in the general area of digital communication theory and optical communication systems, with special attention to adaptive optical and electronic equalization, channel coding, and advanced modulation formats for optical systems. He is the leader of the "Optical Transmission Theory and Techniques" area at the Integrated Research Center for Photonic Networks and Technologies (IRCPhoNeT), Pisa, Italy. His research activity has led to numerous scientific publications in leading international journals and conference proceedings, as well as a few patents. In 2004 he was General Chairman of the Tyrrhenian International Workshop on Digital Communications.

Giancarlo Prati (M'81-F'03) was born in Rome, Italy, on November 13, 1946. He received the Dr. Ing. degree in electronics engineering (cum laude) from the University of Pisa, Pisa, Italy, in 1972.

From 1975 to 1978 he was Associate Professor of Electrical Engineering at the University of Pisa. From 1978 to 1979 he was on a NATO-supported Fellowship Leave in the Department of Electrical Engineering, University of Southern California, Los Angeles working in optical communications. In 1982 he was Visiting Associate Professor in the Department of Electrical and Computer Engineering, University of Massachusetts, Amherst. From 1976 to 1986 he was a Research Scientist of the Italian National Research Council (CNR) at the Centro di Studio per Metodi e Dispositivi di Radiotrasmissione, Pisa. From 1986 to 1988 he was Professor of Electrical Engineering at the University of Genoa, Italy. From 1988 to 2000 he was Professor of Telecommunications Engineering at the University of Parma, Italy, where he served as Dean of the Engineering Faculty from 1992 to 1998 . He is now Professor of Telecommunications at the Scuola Superiore S. Anna in Pisa, Italy. Currently he is President of CNIT, Italian Interuniversity Consortium for Telecommunications, incorporating 35 Universities. From 1997 to 2006 he was a Member of the Technical Program Committee of the European Conference on Optical Communications (ECOC). His professional and academic interests are in telecommunication systems and digital signal processing in communications. The activity has focused on optical communications and radiofrequency communications, with application to satellite communications, high-capacity terrestrial digital radio links, mobile radio, modems for switched telephone lines, fiber communications. 